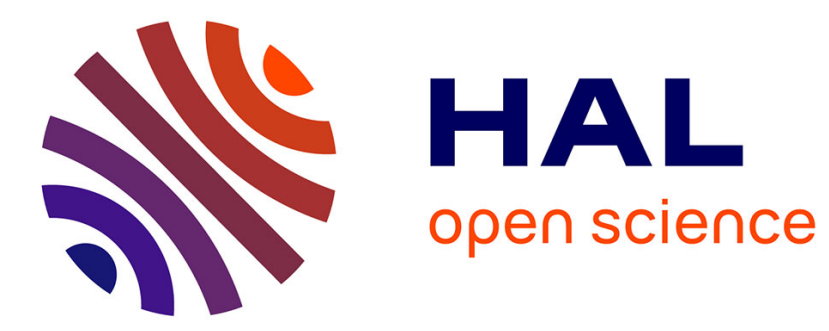

\title{
Definition of a new predictor for multiaxial fatigue crack nucleation in rubber
}

Erwan Verron, Andri Andriyana

\section{To cite this version:}

Erwan Verron, Andri Andriyana. Definition of a new predictor for multiaxial fatigue crack nucleation in rubber. Journal of the Mechanics and Physics of Solids, 2008, 56 (2), pp.417-443. 10.1016/j.jmps.2007.05.019 . hal-01006719

\section{HAL Id: hal-01006719 https://hal.science/hal-01006719}

Submitted on 16 Jun 2014

HAL is a multi-disciplinary open access archive for the deposit and dissemination of scientific research documents, whether they are published or not. The documents may come from teaching and research institutions in France or abroad, or from public or private research centers.
L'archive ouverte pluridisciplinaire HAL, est destinée au dépôt et à la diffusion de documents scientifiques de niveau recherche, publiés ou non, émanant des établissements d'enseignement et de recherche français ou étrangers, des laboratoires publics ou privés. 


\title{
Definition of a new predictor for multiaxial fatigue crack nucleation in rubber
}

\author{
E. Verron ${ }^{\mathrm{a}}$, A. Andriyana ${ }^{\mathrm{b}}$ \\ ${ }^{a}$ Institut de Recherche en Génie Civil et Mécanique, UMR CNRS 6183, Ecole Centrale de Nantes, BP 92101, 44321 Nantes cedex 3, France \\ b LAMEFIP, Ecole Nationale Supérieure d'Arts et Métiers, 33405 Talence, France
}

From an engineering point of view, prediction of fatigue crack nucleation in automotive rubber parts is an essential prerequisite for the design of new components. We have derived a new predictor for fatigue crack nucleation in rubber. It is motivated by microscopic mechanisms induced by fatigue and developed in the framework of Configurational Mechanics. As the occurrence of macroscopic fatigue cracks is the consequence of the growth of pre-existing microscopic defects, the energy release rate of these flaws need to be quantified. It is shown that this microstructural evolution is governed by the smallest eigenvalue of the configurational (Eshelby) stress tensor. Indeed, this quantity appears to be a relevant multiaxial fatigue predictor under proportional loading conditions. Then, its generalization to non-proportional multiaxial fatigue problems is derived. Results show that the present predictor, which is related to the previously published predictors, is capable to unify multiaxial fatigue data.

Keywords: Energy release rate; Crack mechanics; Finite strain; Rubber material; Configurational mechanics

\section{Introduction}

The last decade experienced major advances in the development of finite element tools for the simulation of a wide range of industrial rubber parts. The corresponding studies were mainly motivated by the need to improve time and cost efficiencies in the automotive industry; they are especially devoted to anti-vibration systems (AVS) such as vibration isolators, structural bearings, ... . While actual simulation tools satisfactorily predict stress and strain histories of rubber parts under service loading conditions, the use of these results to estimate the fatigue life of components remains a critical issue.

As emphasized by Mars and Fatemi (2002), two approaches are generally adopted to define the end-of-life of rubber parts: crack nucleation and crack growth approaches. While crack growth approach has been extensively investigated since the pioneering work of Rivlin and Thomas (1953), less attention has been given to crack nucleation approach despite its simplicity (see Mars and Fatemi, 2002 and the references herein). 
In fact, the latter is advantageous to predict the spatial distribution of fatigue life in ideal parts, i.e. without macroscopic defect, as it relates the fatigue life to the history of quantities defined at material points in the sense of continuum mechanics. Therefore, this approach can be used during the product development process in order to reduce the number of fatigue experiments.

In the industrial context, AVS end-of-life is defined by a significant decrease in their mechanical stiffness. Previous studies showed that this decrease can be correlated with the number of cycles required to cause the appearance of a crack of a certain size (Cadwell et al., 1940; Fielding, 1943; ASTM, 1994; Mars, 2001). Thus, experimentally, both stiffness decrease and crack occurrence can be used to define samples end-of-life. In order to relate experimental measurement of the end-of-life with numerical results, a relevant continuum mechanics quantity should be defined. Then, for given loading conditions, the numerical values of this quantity are plotted against the end-of-life, i.e. number of cycles to failure, to obtain the so-called Wöhler curve. This mechanical quantity is referred to as a predictor through the rest of the paper. Then, a fatigue life criterion can be obtained by comparing this predictor with its critical value, i.e. its experimental value at end-of-life for a given material.

In our opinion, a relevant predictor should fulfil the following conditions:

(i) it should be written in terms of continuum mechanics quantities rather than fracture mechanics quantities in order to predict the spatial distribution of fatigue life in each particle of a rubber body without macroscopic defect (see above),

(ii) its formulation should be motivated by the physical phenomena observed during fatigue crack nucleation experiments. Thus, the microscopic mechanisms representative of fatigue damage should be considered to derive the predictor,

(iii) it should be theoretically well formulated,

(iv) it should be easily implementable into finite element softwares without requiring excessive running time.

Finally, in order to validate the theoretical derivation, the multiaxial ability of the predictor has to be demonstrated: for different loading conditions (for example uniaxial tension, equibiaxial tension, torsion, shear, ...), the predictor should be able to unify experimental end-of-life data, i.e. the corresponding Wöhler curves have to be superimposed.

The aim of the present paper is to develop such a predictor. More precisely, the emphasis will be laid on requirements (ii) and (iii) because requirement (i) is considered as an essential prerequisite. Requirement (iv) will be examined in further work. It is to note that no fatigue life criterion will be proposed here; such proposal necessitates the determination of the critical value of the predictor by means of appropriate experimental measurements. This value will depend on the considered material.

The paper is organized as follows. In Section 2, microscopic mechanisms involved during fatigue crack nucleation in rubber will be first presented; the importance of pre-existing flaws in component life will be highlighted and the necessity to consider their energy release rate will be demonstrated. Then this section will be closed by the presentation of the classical predictors adopted for rubber fatigue life. Section 3 is devoted to the derivation of the predictor. First, it will be shown that the general theory of Configurational Mechanics, initially introduced by Eshelby $(1951,1975)$ is the appropriate tool to rationalize previously published works. So, a brief overview of this theory will be given. Then, the derivation of our predictor is detailed by considering both simple proportional and complex multiaxial loading conditions. The validation of our approach is presented in Section 4. First, basic analytical results are established. Then, previously published experimental data are considered to demonstrate the efficiency of the predictor. Finally, Section 5 closes the paper.

\section{Physical motivation and classical predictors}

\subsection{Microstructural mechanisms}

From a macroscopic point of view, fatigue crack nucleation is generally defined by the occurrence of a small crack of a given size (1 mm for André et al., 1999; $2 \mathrm{~mm}$ for Ostoja-Kuczynski et al., 2003). Such cracks usually 
correspond with a decrease of the component stiffness. It has been believed for a long time that crack nucleation observed at the macroscopic scale is the consequence of the propagation of microscopic defects presumably present in the virgin material (Gent et al., 1964). Typical flaws found in rubber are carbon black agglomerates, compounding inclusions and cavities; they are thought to be equally distributed throughout the material. Therefore, the understanding of macroscopic fatigue crack initiation necessitates the study of both nucleation and propagation of microscopic flaws.

Two main mechanisms were found to occur during microscopic fatigue crack nucleation independently of loading conditions: decohesion between rubber matrix and inclusions, and cavitation (Le Cam, 2005; Saintier et al., 2006). Decohesion was mostly observed at rigid inclusions such as $\mathrm{SiO}_{2}$ or $\mathrm{CaCO}_{3}$ while cavitation, the spontaneous process of void nucleation, was found to occur in the neighbourhood of carbon black agglomerates. Even though flaws of all orientations are presumed to be initially present, the growth of favourably oriented flaws with respect to loading conditions is more rapid than other flaws (Mars and Fatemi, 2002). Concerning propagation of flaws, Le Cam et al. (2004) recently demonstrated that fatigue crack propagation in filled natural rubber can be explained by growth and coalescence of small voids being predominantly the consequence of the decohesion between rubber matrix and zinc oxides or filler aggregates. These observations generalize previous studies which highlighted the influence of cavitation on crack initiation and propagation in elastomers (see for example the works of Gent, 1990 for static loading conditions and of Legorju-Jago and Bathias, 2002 for fatigue under high hydrostatic pressure).

Finally, to emphasize the importance of flaw growth on macroscopic fatigue crack nucleation, two recent works can be cited. In his Ph.D. thesis, Le Cam (2005) demonstrates that flaws grow from the very first loading cycle. Moreover, it was also shown that about $75 \%$ of the fatigue life of the part (defined in this study by the number of cycles required to create a $1 \mathrm{~mm}$ long crack on a diabolo sample) corresponds to the propagation of pre-existing flaws. More recently, Hainsworth (2007) observed small cracks in sample edges after cycling to $8 \%$ of the fatigue life, and the emergence of a dominant crack at the specimen edge at $40 \%$ of the life.

\subsection{Existing predictors for fatigue crack nucleation}

The crack nucleation approach, which follows the work of Wöhler (1867) and was first applied to rubber by Cadwell et al. (1940), considers that fatigue life of rubbers can be determined from the history of strain and stress at each material point in the body. The three most widely used predictors for rubber fatigue are the maximum stretch ratio, the maximum principal Cauchy stress and the strain energy density. They are briefly presented in the following sections. Then, the recent predictor, the cracking energy density proposed by Mars (2002), which attempts to encompass microscopic observations, is presented.

\subsubsection{Maximum stretch ratio $\left(\lambda_{\max }\right)$}

As most of rubber fatigue experiments are conducted by prescribing certain displacement to specimens, it is considered quite natural to relate fatigue life to strain measures. Furthermore, it was widely observed that cracks initiate in a plane normal to the maximum tensile strain. Earliest studies investigated fatigue life of rubber for different stretch levels in both uniaxial extension and shear; they showed that for elastomers which crystallize under strain (such as natural rubber), increasing minimum stretch can significantly improve the fatigue life (Cadwell et al., 1940; Fielding, 1943). More recently, Roberts and Benzies (1977) and Roach (1982) investigated fatigue life under conditions of simple and equibiaxial tension: when plotted against the maximum stretch ratio (or strain), fatigue life was longer in simple tension than in equibiaxial tension. Several years later, Ro (1989) concluded that strain is generally not relevant for unifying multiaxial fatigue data.

\subsubsection{Maximum principal Cauchy stress $\left(\sigma_{\max }\right)$}

Bathias et al. (1998), André et al. (1999), Abraham et al. (2005), Saintier et al. (2006) correlated fatigue life with the maximum principal Cauchy stress. In fact, the authors adopted this predictor in order to investigate fatigue life improvement due to crystallization in rubber under non-relaxing tension cyclic loading condition: in general, for constant maximum stress level, fatigue life is observed to increase when the minimum stress is strictly positive. Under simple torsion, André et al. (1999) showed that cracks orientation is perpendicular to 
the direction of the largest principal Cauchy stress; consequently, they suggested that this predictor may be the appropriate local variable to describe multiaxial fatigue damage. Nevertheless, Abraham et al. (2005) inferred that the maximum principal Cauchy stress cannot be used to predict fatigue life of elastomers.

\subsubsection{Strain energy density $(W)$}

After the development of fracture mechanics for rubber in the early 1960s, strain energy density came into use as a parameter to predict fatigue crack nucleation because under simple loading conditions the energy release rate is proportional to the product of strain energy density and crack size (Gent et al., 1964). Therefore, the strain energy density can be considered as a measure of the energy release rate of pre-existing flaws in relation with the phenomena observed during the propagation of microscopic defects. Nevertheless, Roberts and Benzies (1977) and Roach (1982) showed that this predictor is not able to unify Wöhler curves for uniaxial and equibiaxial tension loading conditions. Furthermore, being a scalar quantity, it does not provide a specific orientation of potentially occurring cracks. Despite these observations, Ro (1989) and Abraham et al. (2005) argued that the strain energy density is a better predictor than strain or stress-based predictors.

\subsubsection{Cracking energy density}

The inability of the strain energy density to unify multiaxial fatigue life predictions can be explained by the fact that, in complex problems, only a portion of the strain energy density is available for flaw growth. Approaches that attempt to take into account the energy release rate of microscopic defects in order to render microscopic phenomena were previously investigated by Rivlin and Thomas (1953), Greensmith (1963), Lindley (1972), Young (1990) who related the tearing energy, i.e. the energy release rate of Griffith (1920) as applied to elastomers, to the strain energy density for classical fracture mechanics of rubber test specimens such as the single edge specimen or the trouser specimen. This approach was recently extended to multiaxial loading conditions by Mars (2002) through his concept of cracking energy density. This predictor represents the portion of the total strain energy density that is available to be released as microscopic defects grow. In the context of elasticity, the author postulated that the increment of energy available to be released on a given material plane of normal vector $\mathbf{r}$ is defined as the dot product of the Cauchy traction vector $\boldsymbol{\sigma} \mathbf{r}(\boldsymbol{\sigma}$ being the Cauchy stress tensor) with the increment of strain in the $\mathbf{r}$-direction. This strain increment vector being defined as the product of the increment of the strain tensor $\mathrm{d} \boldsymbol{\varepsilon}$ with the vector $\mathbf{r}$, the increment of energy is given by:

$$
\mathrm{dW}_{c}=(\boldsymbol{\sigma} \mathbf{r}) \cdot(\mathrm{d} \boldsymbol{\varepsilon} \mathbf{r})=\mathbf{r} \cdot \boldsymbol{\sigma} \mathrm{d} \boldsymbol{\varepsilon} \mathbf{r} .
$$

It leads to the definition of an energetic tensor which increment is $\boldsymbol{\sigma} \mathrm{d} \boldsymbol{\varepsilon}$. Then, Mars predictor consists in accumulating this tensor over one cycle and calculating the material plane in which the maximum of energy is released. In the last few years, this theory was used in several papers (Kim et al., 2005; Zine et al., 2006; Martinovs and Gonca, 2006).

\section{Theoretical derivation}

As demonstrated in the previous section, a relevant predictor for rubber fatigue should take into account the fact that macroscopic fatigue crack nucleation is mainly due to the propagation of microscopic defects initially present in rubber. The classical predictors (strain, stress, strain energy) were empirically developed with no connection to the microscopic mechanisms. In our opinion, the concept of cracking energy density proposed by Mars (2002) is more appropriate. However, its theoretical foundation needs to be improved using a well-established theoretical framework. In the following, we consider that Configurational Mechanics, which provides the basis for the study of inhomogeneities of continuous media and for capturing elastic singularities in continuum mechanics (Maugin, 1995), is the relevant theoretical framework to develop a new predictor.

\subsection{Brief overview of Configurational Mechanics}

In this section, the basic concepts and results related to the general theory of Configurational Mechanics are recalled. The emphasis is laid on the definition and the significance of the configurational stress tensor because 
it is the cornerstone of our approach. For more details on the general theory, the reader can refer to the various references cited in the following.

\subsubsection{Basic theory}

The theory of Configurational Mechanics was introduced by Eshelby (1951) when he proposed the concept of energy-momentum tensor and configurational forces in continuum mechanics of solids by studying the driving force of a moving defect. Twenty years later, both Eshelby (1975) and Chadwick (1975) extended the previous theory to finite strain. As the notion of body configuration is essential in this theory, Steinmann (2000) highlighted the importance of its derivation in the finite strain framework.

The Configurational Mechanics is also designated as the Eshelbian Mechanics by Maugin (1993), and the Mechanics in Material Space by Kienzler and Herrmann (2000) by contrast to the Newtonian Mechanics or the Mechanics in Physical Space, respectively. In classical Newtonian Mechanics, attention is focused on physical forces generated by displacements in physical space, i.e. the three-dimensional Euclidean space $\mathscr{E}^{3}$. In Eshelbian Mechanics, we deal with a different class of forces, referred to as configurational forces, which are generated by displacements not in the physical space but in the material space (or manifold) $\mathscr{M}^{3}$, i.e. the abstract set of particles that constitute the body (Truesdell and Noll, 1965). While Gurtin (2000) considers configurational forces as basic objects of new concepts in mechanics, Maugin (1995) shows that they can be directly derived from classical continuum mechanics. Here the latter viewpoint is adopted.

Consider an elastic body in its reference configuration $\left(\mathscr{C}_{\mathrm{R}}\right)$; this configuration is defined in the material manifold $\mathscr{M}^{3}$. Under deformation, the body occupies a series of physical configurations defined in the classical physical space $\mathscr{E}^{3}$. Here, we restrict the discussion to hyperelastic materials characterized by the existence of a strain energy density per unit of volume in the reference configuration $W(\mathbf{F}, \mathbf{X})$. It is considered that the body may be inhomogeneous, i.e. the strain energy density depends on both the deformation gradient and the material position. Considering a static motion under mechanical loading and in absence of body force, balance equations of linear and angular momenta are expressed in the Eulerian description as

$$
\operatorname{div}_{\mathbf{x}} \boldsymbol{\sigma}=\mathbf{0} \text { and } \boldsymbol{\sigma}^{\mathrm{T}}=\boldsymbol{\sigma} .
$$

In this equation, $\mathbf{x}$ is the position of the particle in the deformed configuration, $\boldsymbol{\sigma}$ is the Cauchy stress tensor defined by

$$
\boldsymbol{\sigma}=J^{-1} \frac{\partial W}{\partial \mathbf{F}} \mathbf{F}^{\mathrm{T}}
$$

with $J=\operatorname{det} \mathbf{F}$ and the superscript $\cdot^{\mathrm{T}}$ denoting the transposition. Lagrangian counterparts of the balance laws Eq. (2) are easily derived by expressing the quantities per unit of surface and volume in the reference configuration of the body, it leads to

$$
\operatorname{Div}_{\mathbf{X}} \mathbf{P}=\mathbf{0} \text { and } \quad \mathbf{P F}^{\mathrm{T}}=\mathbf{F} \mathbf{P}^{\mathrm{T}} \text {, }
$$

in which $\mathbf{X}$ is the position of the particle in the reference configuration and $\mathbf{P}$ is the first Piola-Kirchhoff stress tensor given by

$$
\mathbf{P}=\frac{\partial W}{\partial \mathbf{F}} .
$$

Generally, deformation induces microstructural changes or rearrangements in the material, e.g. growth of microscopic defects, dislocation or displacement of boundary phases. As argued by Steinmann (2000), describing such rearrangement in the physical space is not an easy task. Thus, to overcome this difficulty, the balance of physical linear momentum has to be completely written onto the material space (Maugin, 1995); indeed, the pull-back operation that introduced $\mathbf{P}$ from $\boldsymbol{\sigma}$ is only partial because the resulting expression in Eq. (4) is still containing components in the current configuration. So, it is necessary to make one more pullback operation that allows to canonically project balance equations onto the material space $\mathscr{M}^{3}$. For the balance of linear momentum, it can be performed either by applying the Noether's theorem (Gross et al., 2003) or by using a direct method which consists in simply multiplying the left-hand side of Eq. (4) ${ }_{1}$ by $\mathbf{F}^{\mathrm{T}}$ (Maugin, 1995). 
After some algebraic manipulations, this equation reduces to

$$
\operatorname{Div}_{\mathbf{X}} \boldsymbol{\Sigma}+\mathbf{G}=\mathbf{0}
$$

with:

$$
\boldsymbol{\Sigma}=W \mathbf{I}-\mathbf{F}^{\mathrm{T}} \mathbf{P}=W \mathbf{I}-\mathbf{C S}
$$

and

$$
\mathbf{G}=-\left.\frac{\partial W}{\partial \mathbf{X}}\right|_{\text {expl }} .
$$

In Eq. (7), $\boldsymbol{\Sigma}$ is the configurational stress tensor (or the energy momentum tensor or the Eshelby stress tensor) and $\mathbf{I}$ is the $3 \times 3$ identity tensor. In Eq. (7), $\mathbf{S}$ is the second Piola-Kirchhoff stress tensor and $\mathbf{C}$ is the right Cauchy-Green strain tensor equal to $\mathbf{F}^{\mathrm{T}} \mathbf{F}$. Moreover, in Eq. (8) $\mathbf{G}$ is referred to as the configurational force vector associated with inhomogeneities. It is defined by the negative explicit differentiation of the strain energy with respect to the particle position in the material manifold (see the index $\left.\cdot\right|_{\text {expl }}$ ). Considering the balance of physical angular momentum, the configurational stress tensor satisfies the following symmetry condition (Epstein and Maugin, 1990):

$$
\boldsymbol{\Sigma} \mathbf{C}=\mathbf{C} \boldsymbol{\Sigma}^{\mathrm{T}} .
$$

If the material is assumed homogeneous, the configurational force $\mathbf{G}$ vanishes and the configurational stress tensor satisfies a strict conservation law

$$
\operatorname{Div}_{\mathbf{X}} \boldsymbol{\Sigma}=\mathbf{0} \text {. }
$$

Moreover, in the special case of isotropic materials, for which $\mathbf{C}$ and $\mathbf{S}$ are coaxial and then commute, Eq. (9) simplifies and the configurational stress tensor is symmetric.

\subsubsection{Applications}

In the majority of studies involving Configurational Mechanics, only configurational forces are investigated through the calculation of path-independent integrals around inhomogeneities, because these forces are recognized as the driving forces of defect evolution. So, configurational stress only appears in the definition of surface tractions, i.e. after contraction with the outward normal of the contour. Most of these works focus on the application of Configurational Mechanics to Fracture Mechanics (see for example Steinmann, 2000 and the references herein). Indeed, it is now well-established that, for hyperelastostatic problems, configurational forces which apply on crack tip generalize the classical J-(Rice, 1968), L- and M-(Budiansky and Rice, 1973) path-independent integrals. Extensions for fracture of more complex materials are proposed by Maugin et al. (1992) for electromagnetism, by Maugin (1999) for thermoelasticity, by Liebe et al. (2003) for continuum damage and by Nguyen et al. (2005) for linear viscoelasticity and elastoplasticity. Moreover, as proposed by Kolling et al. (2003), other problems can be analysed with the help of the Configurational framework: dislocations in metal (Steinmann, 2002), movement of interfaces in two-phase bodies (Gurtin, 2000), defects in piezo-electric material (Gross et al., 2003), etc. Finally, numerical procedures devoted to configurational forces were recently examined by Steinmann et al. (2001), Denzer et al. (2003) and Menzel et al. (2004).

Opposite to the case of configurational forces, only few studies are concerned with the peculiar properties of the configurational stress tensor $\boldsymbol{\Sigma}$. As an example, for the linear theory, the physical significance of the Cartesian components of this tensor were identified only recently by Kienzler and Herrmann (1997), who explain that "[the $i j$-component] of the Eshelby tensor is the change in the total energy density at a point of an elastic continuum due to a material unit translation in $x_{j}$ direction of a unit surface with normal in $x_{i}$ direction". Authors also investigated principal values and directions of the tensor in two dimensions. More recently, they introduced local fracture criteria for small strain problems based on the components of the configurational stress tensor (Kienzler and Herrmann, 2002): in two dimensions, principal values and a von Mises-like value of this tensor were related to the classical stress intensity factors of linear fracture mechanics. Until now, we are not aware of other accounts which explicitly deal with the components of the configurational stress tensor. 
Remark 1. The previous definition of $\sum_{i j}$ can be extended to the finite strain framework without restriction (Andriyana, 2006). This tensor being completely defined in the material manifold, one should consider a unit surface in the reference configuration and moves this surface by a unit material translation, i.e. a translation in this manifold. So, $\boldsymbol{\Sigma}$ contains energy changes due to the evolution of the reference configuration (or the change of natural configuration as proposed by Rajagopal and Srinivasa, 2005), i.e. a continuum expression of microstructural evolution. As an example, the growth of a straight crack from a length $a$ to $a+d a$ is such a material translation.

In fact, considering the geometrical definition via the notion of local structural rearrangement advocated by Epstein and Maugin (1990), it is to say that the configurational stress is the driving force governing local structural rearrangements (Maugin, 2002). As fatigue loading conditions induce significant microstructural rearrangements in rubber, it becomes obvious that configurational stresses, in the appropriate form, are the driving forces of these evolutions (Andriyana, 2006; Andriyana and Verron, 2007). Thus, based on the above theoretical summary and considering the physical significance of the configurational stress components as proposed by Kienzler and Herrmann (1997), we consider that the Eshelby stress tensor, which focuses on the behaviour of defects in the material manifold, appears to be the relevant continuum mechanics quantity to derive a new fatigue life predictor for rubber.

\subsection{Development of the predictor}

In the following, rubber is assumed to be hyperelastic, isotropic and incompressible. Only isothermal processes are investigated. Moreover, the material is considered homogeneous at the scale of the continuum. This mesoscopic scale is defined by the representative volume element (RVE) in which material properties can be represented by continuous fields: mass density, stress, strain, ... (Lemaitre, 2001). Indeed, for elastomers, the RVE contains rubber matrix and a large number of microscopic defects such as carbon black agglomerates, inclusions, cavities, ... (Mars and Fatemi, 2002; Le Cam et al., 2004). The transition between the microscopic and the mesoscopic scales will be the foundation of our theory. So, with the previous assumptions, the mechanical response of the material can be entirely defined by a strain energy density $W$ which depends on the two first strain invariants:

$$
W=\widetilde{W}\left(I_{1}, I_{2}\right)
$$

with

$$
I_{1}=\operatorname{tr} \mathbf{C} \quad \text { and } \quad I_{2}=\frac{1}{2}\left[(\operatorname{tr} \mathbf{C})^{2}-\operatorname{tr}\left(\mathbf{C}^{2}\right)\right] .
$$

The third strain invariant $I_{3}=\operatorname{det} \mathbf{C}$ is equal to 1 due to the incompressibility assumption. For details on hyperelasticity and large strain, the reader can refer to the monograph of Holzapfel (2000).

Consider a rubber body $\mathscr{B}$ defined by its reference configuration $\left(\mathscr{C}_{\mathrm{R}}\right)$. This configuration is defined as a set of particles in the material manifold $\mathscr{M}^{3}$; it is depicted in Fig. 1. One particle of this set is located at $\mathbf{X}$ in the physical space $\mathscr{E}_{3}$. To simplify the discussion, the reference configuration is assumed to correspond to a vanishing stress and strain, stable configuration of minimum energy. Under mechanical loading, the body deforms and occupies a time sequence of physical configurations (in $\left.\mathscr{E}^{3}\right)$. Let $(\mathscr{C})$ be the body configuration at time $t$ defined by the mapping $\mathbf{x}(\mathbf{X}, t)$ and by its gradient $\mathbf{F}(\mathbf{X}, t)$. As shown in Fig. 1, when loading is removed the body will, in general, occupy a new stress-free configuration $\left(\mathscr{C}_{\mathrm{R}}^{\prime}\right)$ defined by the motion gradient $\mathbf{f}(\mathbf{x}, t)$. Both configurations $\left(\mathscr{C}_{\mathrm{R}}\right)$ and $\left(\mathscr{C}_{\mathrm{R}}^{\prime}\right)$ represent natural configurations of the body (Rajagopal and Srinivasa, 2005). The physical reasons for a body to possess different natural configurations can be very diverse. Indeed, it is the consequence of structural rearrangements at the microscopic scale, e.g. movement of dislocations, cavitation, cleavage fracture, which manifests in various ways at the mesoscopic scale, e.g. plasticity, damage; or at the macroscopic scale, e.g. crack growth. If the material is perfectly elastic, $\mathbf{f}=\mathbf{F}^{-1}$ and the natural configurations $\left(\mathscr{C}_{\mathrm{R}}\right)$ and $\left(\mathscr{C}_{\mathrm{R}}^{\prime}\right)$ are identical. Nevertheless, if irreversible microstructural changes take place during the motion, the gradient $\mathbf{f}$ can be considered close, but not equal, to $\mathbf{F}^{-1}$.

To illustrate this discrepancy, we focus our attention on the deformation of the RVE. Consider a given material point $P$ of the body $\mathscr{B}$. The motion of the RVE which constitutes the particle $P$ is shown in Fig. 2. 


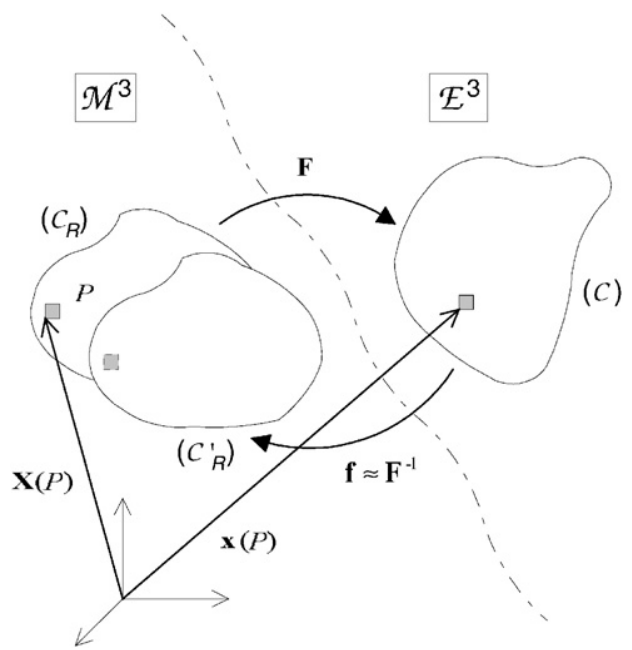

Fig. 1. Motion of a rubber body $\mathscr{B}$. Definition of its configurations.

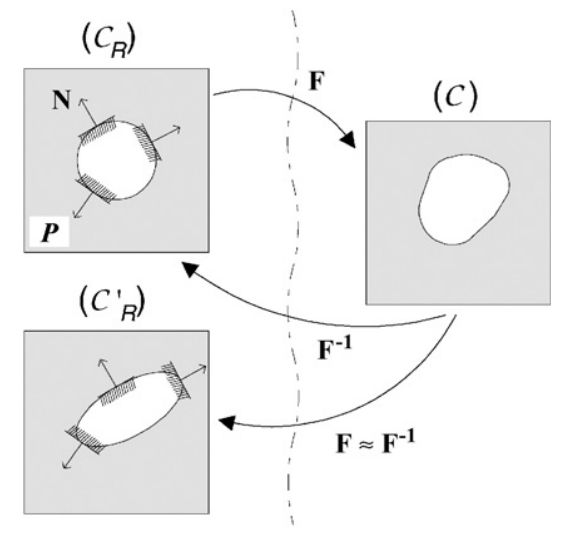

Fig. 2. Motion of the RVE $P$ at the microscopic scale.

This RVE contains both bulk material (grey in the figure) and various defects which are schematized by the white circle in the figure. Under motion, the defect evolves: it may grow or shrink as shown in the deformed configuration $(\mathscr{C})$ in Fig. 2. Note that the size and shape of the RVE, i.e. the grey square, remain unchanged during motion because it is considered that microstructural rearrangements do not change the definition of the RVE. Then, similarly to Fig. 1, if the rearrangement is reversible, the defect will recover its initial size and shape defined by $\left(\mathscr{C}_{\mathrm{R}}\right)$ after unloading; otherwise the microstructural change is irreversible. Consequently, the defect configuration evolves as shown in the configuration $\left(\mathscr{C}_{\mathrm{R}}^{\prime}\right)$ of Fig. 2. It might be confusing to consider irreversible process, i.e. dissipation, in an elastic material. However, as underlined by Gross et al. (2003), the presence of microscopic defects in the body yields to the change of its total energy if these defects move relatively to the reference configuration. So, recalling the microstructural phenomena observed in rubber under fatigue loading conditions and presented above, we should now quantify the energy released during this structural rearrangement, i.e. calculate the energy release rate between configurations $\left(\mathscr{C}_{\mathrm{R}}\right)$ and $\left(\mathscr{C}_{\mathrm{R}}^{\prime}\right)$ depicted in Fig. 2.

As emphasized above, energetic properties of microstructural rearrangements are completely encompassed within the configurational stress tensor $\boldsymbol{\Sigma}$. Thus, as this stress tensor is the driving force governing local structural rearrangement (Maugin, 2002), the evolution of the flaw between $\left(\mathscr{C}_{\mathrm{R}}\right)$ and $\left(\mathscr{C}_{\mathrm{R}}^{\prime}\right)$ is driven by this tensor. As depicted in Fig. 3, consider a unit material surface of outward normal vector $\mathbf{N}$ on the flaw boundary (see also Fig. 2). Due to the deformation defined by the gradient $\mathbf{F}$, this surface is subjected to the 


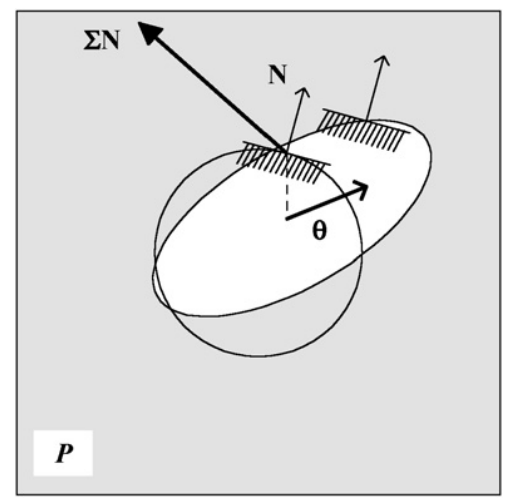

Fig. 3. Evolution of the flaw.

material traction $\boldsymbol{\Sigma} \mathbf{N}$. Recalling the definition of the components of $\boldsymbol{\Sigma}$ proposed by Kienzler and Herrmann (1997) (see Section 3.1.2), for a given material translation $\boldsymbol{\theta}$ of the previous surface, i.e. a translation in the material manifold (not a physical one), the scalar $\boldsymbol{\theta} \cdot \mathbf{\Sigma N}$ represents the change of total energy at the material point $P$ due to the change of configuration of the defect between the natural configurations $\left(\mathscr{C}_{\mathrm{R}}\right)$ and $\left(\mathscr{C}_{\mathrm{R}}^{\prime}\right)$. Knowing that the body will tend to reduce as much as possible its total energy during microstructural evolution, one should determine the material surface defined by $\mathbf{N}$ and the material translation $\boldsymbol{\theta}$ which maximize the energy release rate. Moreover, as energy changes are defined positively in $\boldsymbol{\Sigma}$, material vectors $\mathbf{N}$ and $\boldsymbol{\theta}$ which maximize $\boldsymbol{\theta} \cdot(-\boldsymbol{\Sigma}) \mathbf{N}$ should be considered. Note that this is similar to the definition proposed by Eshelby $(1951,1975)$ for the force on a defect which is equal to the negative gradient of the total energy with respect to the change in position of the defect; and with the remark of Steinmann et al. (2001) who consider that the morphology of defects evolves with respect to the direction opposite to the material force.

Considering experimental observations of fatigue damage in rubbers (see Section 2), decohesion and cavitation are the two main mechanisms involved in crack nucleation. Moreover, opening and closing of cavities are supposed to be due to only material normal traction and not due to material shear. Thus, vectors $\mathbf{N}$ and $\boldsymbol{\theta}$ coincide with the principal directions of $-\boldsymbol{\Sigma}$ and more precisely with the principal direction associated with its maximum eigenvalue, i.e. the minimum principal configurational stress. Finally, the fatigue life predictor, denoted $\Sigma^{*}$, can be written as

$$
\Sigma^{*}=\left|\min \left(\left(\Sigma_{i}\right)_{i=1,2,3}, 0\right)\right|,
$$

where $\left(\sum_{i}\right)_{i=1,2,3}$ are the principal configurational stresses. When one (or more) principal stress is negative, the predictor is strictly positive, and the defect tends to grow and to turn into a plane crack orthogonal to $\mathbf{V}^{*}$, the eigenvector associated with $-\Sigma^{*}$. When the three principal stresses are positive, the material tractions tend to shrink the flaw. In this case, the predictor is set to 0 ; it can be seen as the microscopic counterpart of the classical crack-closure effect.

Remark 2. In the hyperelastic framework, the second Piola-Kirchhoff stress tensor is defined as the differentiation of $W$ with respect to the right Cauchy-Green strain tensor $\mathbf{C}$ (see for example Holzapfel, 2000):

$$
\mathbf{S}=-p \mathbf{C}^{-1}+2 \frac{\partial W}{\partial \mathbf{C}}
$$

where $p$ is an arbitrary scalar due to the incompressibility kinematic constraint. Note that $p$ can only be determined from equilibrium equations and boundary conditions. Moreover, considering that the strain energy only depends on the two first strain invariants (Eq. (11)), $\mathbf{S}$ reduces to

$$
\mathbf{S}=-p \mathbf{C}^{-1}+2\left(W_{1}+W_{2} I_{1}\right) \mathbf{I}-2 W_{2} \mathbf{C},
$$


where $W_{i}$ are the partial derivatives of the strain energy with respect to the strain invariants:

$$
W_{i}=\frac{\partial \widetilde{W}}{\partial I_{i}} \quad \text { for } i=1,2 .
$$

Thus, considering Eqs. $(7)_{2}$ and (15), the configurational stress tensor can be written as:

$$
\boldsymbol{\Sigma}=(W+p) \mathbf{I}-2\left(W_{1}+I_{1} W_{2}\right) \mathbf{C}+2 W_{2} \mathbf{C}^{2} .
$$

Remark 3. Recalling that $\mathbf{S}$ and $\mathbf{C}$ are coaxial for isotropic hyperelastic materials and denoting $\left(\widehat{\mathbf{N}}_{i}\right)_{i=1,2,3}$ the principal strain directions in the reference configuration (also called principal referential directions) these tensors can be written as:

$$
\mathbf{C}=\sum_{i=1}^{3} \lambda_{i}^{2} \widehat{\mathbf{N}}_{i} \otimes \widehat{\mathbf{N}}_{i} \quad \text { and } \quad \mathbf{S}=\sum_{i=1}^{3} S_{i} \widehat{\mathbf{N}}_{i} \otimes \widehat{\mathbf{N}}_{i}
$$

where $\left(\lambda_{i}\right)_{i=1,2,3}$ and $\left(S_{i}\right)_{i=1,2,3}$ are respectively the stretch ratios and the principal second Piola-Kirchhoff stresses. Thus, the configurational stress tensor reduces to:

$$
\boldsymbol{\Sigma}=W \mathbf{I}-\sum_{i=1}^{3} \lambda_{i}^{2} S_{i} \widehat{\mathbf{N}}_{i} \otimes \widehat{\mathbf{N}}_{i}=\sum_{i=1}^{3}\left(W-\lambda_{i}^{2} S_{i}\right) \widehat{\mathbf{N}}_{i} \otimes \widehat{\mathbf{N}}_{i}
$$

Moreover, as the principal Cauchy stress are related to the principal second Piola-Kirchhoff stress by

$$
\sigma_{i}=\lambda_{i}^{2} S_{i} \text { for } i=1,2,3,
$$

the configurational stress tensor can also be written as

$$
\boldsymbol{\Sigma}=\sum_{i=1}^{3}\left(W-\sigma_{i}\right) \widehat{\mathbf{N}}_{i} \otimes \widehat{\mathbf{N}}_{i}
$$

However, it should be noted that the quantity $\sum_{i=1}^{3} \sigma_{i} \widehat{\mathbf{N}}_{i} \otimes \widehat{\mathbf{N}}_{i}$ in the right-hand side of this equation is not the Cauchy stress tensor; indeed the spectral decomposition of the Cauchy stress tensor is

$$
\boldsymbol{\sigma}=\sum_{i=1}^{3} \sigma_{i} \widehat{\mathbf{n}}_{i} \otimes \widehat{\mathbf{n}}_{i}
$$

where $\left(\widehat{\mathbf{n}}_{i}\right)_{i=1,2,3}$ are the principal spatial directions defined in the deformed configuration $(\mathscr{C})$ and related to $\left(\widehat{\mathbf{N}}_{i}\right)_{i=1,2,3}$ by the rotation tensor issued from the polar decomposition (Holzapfel, 2000, p. 90).

Remark 4. Examining the previous Eq. (21) proves that there exist relationships between the proposed predictor $\Sigma^{*}$ and the three classical predictors:

- For $W$ it is obvious.

- In regard to Eqs. (13) and (21), the predictor reduces to

$$
\Sigma^{*}=\max \left(\sigma_{\max }-W, 0\right),
$$

where $\sigma_{\max }$ is the maximum principal Cauchy stress. Thus, the new predictor is related to the classical maximum principal stress predictor.

- Finally, if $\sigma_{\max }>W$ in Eq. (23) then

$$
\Sigma^{*}=\sigma_{\max }-W
$$

and the normal vector to the crack $\mathbf{V}^{*}$ is the principal referential direction associated with the principal strain which corresponds to $\sigma_{\max }$. In fact, considering the Baker and Ericksen (1954) inequality,

$$
\frac{\sigma_{i}-\sigma_{j}}{\lambda_{i}-\lambda_{j}}>0 \quad \text { with } \lambda_{i} \neq \lambda_{j}
$$

which states that the principal Cauchy stresses have the same ordering as the stretch ratios, the normal vector to the crack plane is the principal referential direction corresponding to the maximum stretch ratio $\lambda_{\max }$. In this way, our predictor is also related to the maximum strain predictor. 
Remark 5. Note that in the present case of isotropic hyperelasticity, $\boldsymbol{\Sigma}$ is symmetric and then admits three real eigenvalues; the more complex case of viscoelasticity in which $\boldsymbol{\Sigma}$ may be not symmetric is partially discussed in Andriyana (2006).

\subsection{Extension to multiaxial fatigue}

The previous predictor defines an instantaneous value of fatigue damage in every material points of the body, but it is not able to take into account deformation history. Two different time scales should be considered to extend this predictor to multiaxial problems. The first time scale is the one of the loading cycle, i.e. the cycle period. Classically, the derivation of multiaxial fatigue predictors over one cycle is achieved by using quantities defined in the cycle such as stress or strain or energy amplitudes, mean values, maximum values, ... The second time scale is the one of the fatigue life, defined by numbers of cycles. In the present work, we only focus on the former time scale, the latter will be investigated in further studies. As elastomers exhibit a steady state cyclic response under fatigue loading conditions, characterized by a stabilized hysteretic stress-strain response (André et al., 1999; Abraham et al., 2005), the determination of the fatigue predictor over only one stabilized cycle is supposed sufficient to predict fatigue end-of-life. It is to note that the determination of this appropriate stabilized cycle is not an easy task.

Thus, to take into account the deformation history, the cumulation of fatigue damage should be performed over one loading cycle. More precisely, only the part of the configurational stress tensor which contributes to flaw opening should be accumulated. This part will be referred to as the damage part of the configurational stress tensor and denoted $\boldsymbol{\Sigma}^{\mathrm{d}}$ in the following. Let $\mathrm{d} \boldsymbol{\Sigma}^{\mathrm{d}}$ be the increment of $\boldsymbol{\Sigma}^{\mathrm{d}}$, thus

$$
\boldsymbol{\Sigma}^{\mathrm{d}}=\int_{\text {cycle }} \mathrm{d} \boldsymbol{\Sigma}^{\mathrm{d}}
$$

Actually, $\mathrm{d} \boldsymbol{\Sigma}^{\mathrm{d}}$ is the damage part of the increment of the configurational stress tensor $\mathrm{d} \boldsymbol{\Sigma}$ which is defined by

$$
\mathrm{d} \boldsymbol{\Sigma}=\frac{\mathrm{d} \boldsymbol{\Sigma}}{\mathrm{d} \mathbf{C}}: \mathrm{d} \mathbf{C},
$$

because $\boldsymbol{\Sigma}$ only depends on the strain tensor $\mathbf{C}$ for hyperelastic materials. To relate $\mathrm{d} \boldsymbol{\Sigma}^{\mathrm{d}}$ to $\mathrm{d} \boldsymbol{\Sigma}$, the previous predictor formula Eq. (13) should be applied by considering the spectral decomposition of the configurational stress increment

$$
\mathrm{d} \boldsymbol{\Sigma}=\sum_{\mathrm{i}=1}^{3} \mathrm{~d} \Sigma_{i} \mathbf{V}_{i} \otimes \mathbf{V}_{i},
$$

where $\left(\mathrm{d} \Sigma_{i}\right)_{i=1,2,3}$ and $\left(\mathbf{V}_{i}\right)_{i=1,2,3}$ are its eigenvalues and eigenvectors respectively. Invoking the same assumption as the one retained for the instantaneous predictor $\Sigma^{*}$, i.e. only normal material tractions are involved in growth of defects, the damage part of $\mathrm{d} \boldsymbol{\Sigma}$ is

$$
\mathrm{d} \boldsymbol{\Sigma}^{\mathrm{d}}=\sum_{\mathrm{i}=1}^{3} \mathrm{~d} \Sigma_{i}^{\mathrm{d}} \mathbf{V}_{i} \otimes \mathbf{V}_{i}
$$

with

$$
\mathrm{d} \Sigma_{i}^{\mathrm{d}}=\left\{\begin{array}{ll}
\mathrm{d} \Sigma_{i} & \text { if } \mathrm{d} \Sigma_{i}<0 \\
0 & \text { otherwise. }
\end{array} \text { and } \mathbf{V}_{i} \cdot \mathbf{\Sigma} \mathbf{V}_{i}<0,\right.
$$

This formulation can be explained as follows:

- For each loading increment, the configurational stress increment $\mathrm{d} \boldsymbol{\Sigma}$ as well as its principal values $\left(\mathrm{d} \Sigma_{i}\right)_{i=1,2,3}$ and principal directions $\left(\mathbf{V}_{i}\right)_{i=1,2,3}$ are computed. Only the part of $\mathrm{d} \boldsymbol{\Sigma}$ that opens defects $\left(\mathrm{d} \Sigma_{i}<0\right)$ is considered.

- To verify the state of defects in a given direction $\mathbf{V}_{i}$, the normal configurational traction that applies in this direction is calculated using the current value of the configurational stress tensor. The defects are said to be in a materially stretched state when $\mathbf{V}_{i} \cdot \Sigma \mathbf{V}_{i}<0$. 
- The components of the fatigue damage configurational stress increment $\mathrm{d} \Sigma_{i}^{\mathrm{d}}$ are defined by retaining only the part of $\mathrm{d} \Sigma$ that opens materially stretched defects, i.e. for each principal directions $\left(\mathbf{V}_{i}\right)_{i=1,2,3}$ when both conditions $\mathrm{d} \Sigma_{i}<0$ and $\mathbf{V}_{i} \cdot \boldsymbol{\Sigma} \mathbf{V}_{i}<0$ are satisfied (Eq. (30)).

Next, the integration of increments over one whole cycle (Eq. (26)) necessitates that all increments are expressed in the same tensorial basis. As eigenvectors of configurational stress increments change in the cycle (for multiaxial loading), this integration should be performed in the global basis adopted to describe the motion

$$
\Sigma^{\mathrm{d}}=\int_{\text {cycle }} \mathrm{d} \Sigma_{k l}^{\mathrm{d}} \mathbf{e}_{k} \otimes \mathbf{e}_{l} .
$$

Finally, the fatigue crack nucleation predictor for multiaxial fatigue loading conditions can be summarized as follows:

Proposition. Multiaxial fatigue predictor for rubber (elastic case):

$$
\Sigma^{*}=\left|\min \left(\left(\Sigma_{i}^{\mathrm{d}}\right)_{i=1,2,3}, 0\right)\right|
$$

where $\left(\Sigma_{i}^{\mathrm{d}}\right)_{i=1,2,3}$ are the eigenvalues of the damage part of the configurational stress tensor $\boldsymbol{\Sigma}^{\mathrm{d}}$. This tensor is obtained by the integration over the cycle of

$$
\mathrm{d} \boldsymbol{\Sigma}^{\mathrm{d}}=\sum_{\mathrm{i}=1}^{3} \mathrm{~d} \Sigma_{i}^{\mathrm{d}} \mathbf{V}_{i} \otimes \mathbf{V}_{i}
$$

with

$$
\mathrm{d} \Sigma_{i}^{\mathrm{d}}=\left\{\begin{array}{ll}
\mathrm{d} \Sigma_{i} & \text { if } \mathrm{d} \Sigma_{i}<0 \\
0 & \text { otherwise, }
\end{array} \text { and } \mathbf{V}_{i} \cdot \mathbf{\Sigma} \mathbf{V}_{i}<0,\right.
$$

$\left(\mathrm{d} \Sigma_{i}\right)_{i=1,2,3}$ and $\left(\mathbf{V}_{i}\right)$ being the eigenvalues and eigenvectors of the configurational stress tensor increment

$$
\mathrm{d} \boldsymbol{\Sigma}=\frac{\mathrm{d} \boldsymbol{\Sigma}}{\mathrm{d} \mathbf{C}}: \mathrm{d} \mathbf{C}
$$

Then,

- if $\Sigma^{*}>0$, the defect tends to grow and to turn into a plane crack orthogonal to $\mathbf{V}^{*}$, the eigenvector corresponding to $-\Sigma^{*}$

- if $\Sigma^{*}=0$, material tractions tend to shrink the flaw.

Remark 6. For fully relaxing proportional loading conditions, the integration over one cycle reduces to the determination of the instantaneous value of the configurational stress tensor for the maximum strain state. In this way, the multiaxial predictor defined in the previous proposition reduces to Eq. (13).

\section{Examples}

In this section, three examples are examined in order to demonstrate the ability of the predictor to satisfactorily estimate multiaxial fatigue damage in rubber. The first example presents the solutions of simple loading problems. The next two ones are comparisons with experimental results published in the bibliography. In these cases, it will be shown that the predictor unifies experimental end-of-life data.

\subsection{Simple deformation of a neo-Hookean material}

This first example compares the results obtained with classical predictors $\lambda_{\max }, \sigma_{\max }$ and $W$ with those obtained with the present predictor for simple loading conditions, i.e. uniaxial extension, pure shear and 
equibiaxial extension. In these cases, as loading conditions are proportional (for equibiaxial loading), Remark 6 applies and the value of $\Sigma^{*}$ reduces to Eq. (13).

In order to simplify the discussion, only the neo-Hookean strain energy density is considered

$$
W=C\left(I_{1}-3\right),
$$

where the material parameter $C$ is set equal to $1 \mathrm{MPa}$. Indeed, $W_{2}=0$ in Eqs. (15) and (17). Thus, the Cauchy and configurational stress tensors are respectively given by

$$
\boldsymbol{\sigma}=-p \mathbf{I}+2 C \mathbf{B}
$$

and

$$
\boldsymbol{\Sigma}=(W+p) \mathbf{I}-2 C \mathbf{C} .
$$

Consider the three simple deformation modes, uniaxial extension, pure shear and equibiaxial extension. In the principal stretching directions $\left(\mathbf{e}_{i}\right)_{i=1,2,3}$, the corresponding deformation gradient can be written in the following compact form:

$$
\mathbf{F}=\lambda \mathbf{e}_{1} \otimes \mathbf{e}_{1}+\lambda^{B} \mathbf{e}_{2} \otimes \mathbf{e}_{2}+\lambda^{-(B+1)} \mathbf{e}_{3} \otimes \mathbf{e}_{3},
$$

where $\lambda$ is referred to as the stretch level and $B$ is the biaxiality factor which takes the following values depending on the deformation mode:

$$
B= \begin{cases}-0.5 & \text { for uniaxial extension, } \\ 0 & \text { for pure shear, } \\ 1 & \text { for equibiaxial extension. }\end{cases}
$$

In the three cases, the body (the sample) being assumed thin in the $\mathbf{e}_{3}$-direction, it is in plane stress conditions and then $\sigma_{33}=0$. This condition is used to determine the incompressibility pressure $p$ in Eqs. (33) and (34); it leads to

$$
\boldsymbol{\sigma}=2 C\left(\lambda^{2}-\lambda^{-2 B-2}\right) \mathbf{e}_{1} \otimes \mathbf{e}_{1}+2 C\left(\lambda^{2 B}-\lambda^{-2 B-2}\right) \mathbf{e}_{2} \otimes \mathbf{e}_{2}
$$

and

$$
\begin{aligned}
\Sigma= & C\left(-\lambda^{2}+\lambda^{2 B}+3 \lambda^{-2(B+1)}-3\right) \mathbf{e}_{1} \otimes \mathbf{e}_{1} \\
& +C\left(\lambda^{2}+\lambda^{2 B}+\lambda^{-2(B+1)}-2 \lambda^{3 B-1}+2 \lambda^{-B-3}-3\right) \mathbf{e}_{2} \otimes \mathbf{e}_{2} \\
& +C\left(\lambda^{2}+\lambda^{2 B}+\lambda^{-2(B+1)}-3\right) \mathbf{e}_{3} \otimes \mathbf{e}_{3} .
\end{aligned}
$$

Thus, for a given stretch level $\lambda$, the classical predictors reduce to

$$
\begin{aligned}
& \lambda_{\max }=F_{11}=\lambda, \\
& \sigma_{\max }=\sigma_{11}=2 C\left(\lambda^{2}-\lambda^{-2 B-2}\right), \\
& W=C\left(\lambda^{2}+\lambda^{2 B}+\lambda^{-2(B+1)}-3\right) .
\end{aligned}
$$

The corresponding results are presented in Figs. 4-6 for uniaxial extension, pure shear and equibiaxial extension respectively. In each figure, left-hand side graph (a) shows the maximum principal Cauchy stress and the strain energy as functions of the stretch level (the maximum stretch predictor is not presented because it is simply equal to the stretch level, see above), and right-hand side (b) shows the three principal configurational stresses. In every cases the predictor is equal to $-\Sigma_{1}$. For uniaxial extension, $\Sigma_{1}$ is the only negative eigenvalue of $\Sigma$ (see Fig. 4(b)) and defects tend to open in the plane normal to $\mathbf{e}_{1}$ as also predicted by stretch and stress predictors. For pure shear, both $\Sigma_{1}$ and $\Sigma_{2}$ are negative with $\left|\Sigma_{1}\right|>\left|\Sigma_{2}\right|$ for $\lambda<1.74$. Then for greater stretch levels, only $\Sigma_{1}$ is negative (see Fig. 5(b)). For all $\lambda$, defects tend to open in the plane normal to $\mathbf{e}_{1}$. Finally, for equibiaxial extension, as shown in Fig. 6(b) $\Sigma_{1}$ and $\Sigma_{2}$ are negative and equal due to the symmetry of loading conditions (it is also similar for $\sigma_{11}$ and $\sigma_{22}$ and for $\lambda_{1}$ and $\lambda_{2}$ ). Indeed, defects may open in planes normal to $\mathbf{e}_{1}$ or $\mathbf{e}_{2}$ with the same probability. 
a

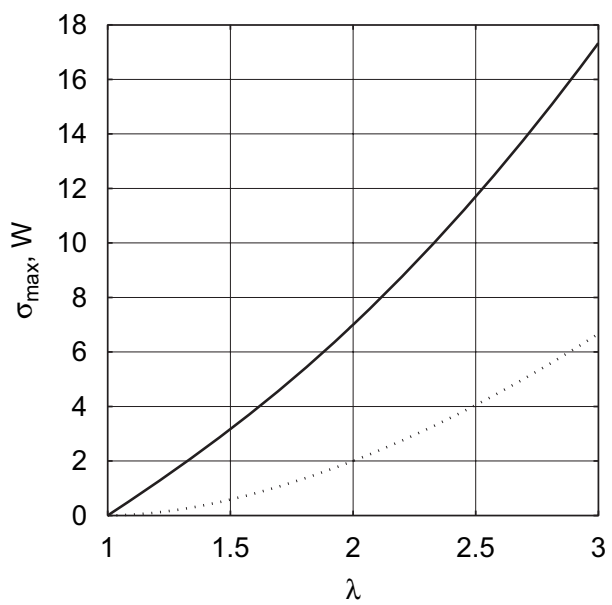

b

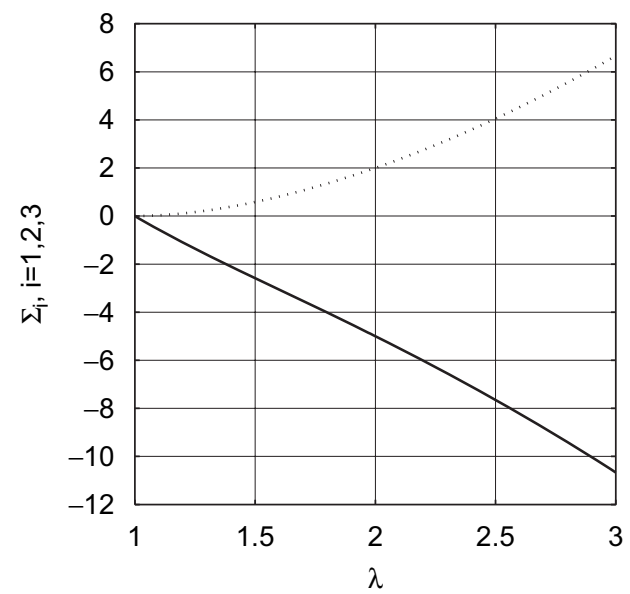

Fig. 4. Uniaxial tensile results. (a) Maximum principal Cauchy stress (-), strain energy ( $\cdots)$, (b) $\Sigma_{1}(-), \Sigma_{2}=\Sigma_{3}(\cdots)$.

a

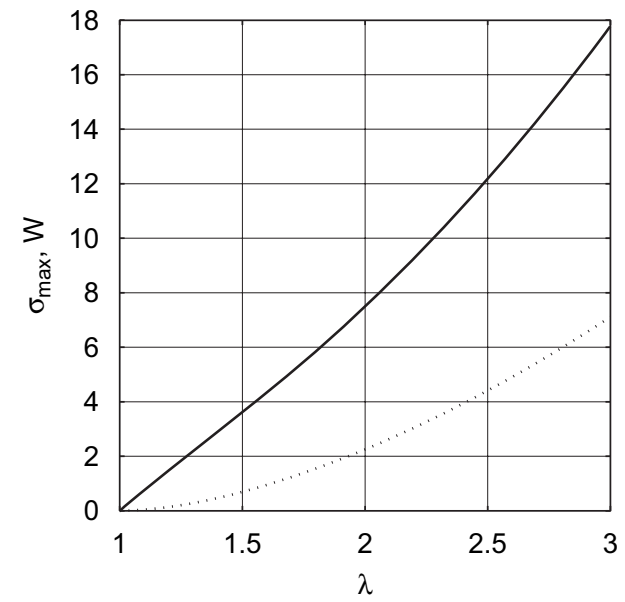

b

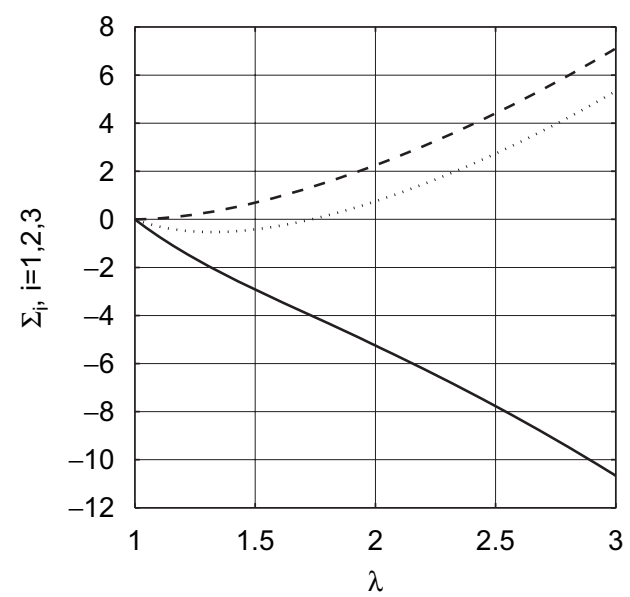

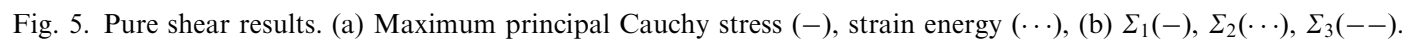

\subsection{Comparison between uniaxial and equibiaxial fatigue prediction}

The aim of this second example is to demonstrate the relevance of our approach by comparing theoretical predictions with multiaxial experimental data. The most different deformation modes for elastomers are the uniaxial and biaxial extension deformation modes: this is the case for the stress-strain response (Ogden, 1972) and also for the fatigue life (Mars, 2002). There are few studies devoted to the comparison between uniaxial and equibiaxial fatigue end-of-life (Gehman and Clifford, 1954; Roberts and Benzies, 1977) because of the difficulty to perform biaxial fatigue experiments. Here we consider the data of Roberts and Benzies (1977), since they are the most comprehensive ones we found in the bibliography.

\subsubsection{Experiments}

In their paper, Roberts and Benzies investigated four elastomers: a carbon black-filled (45 phr) and an unfilled natural rubber (NR), and a carbon black-filled (45 phr) and an unfilled styrene butadiene rubber 
a

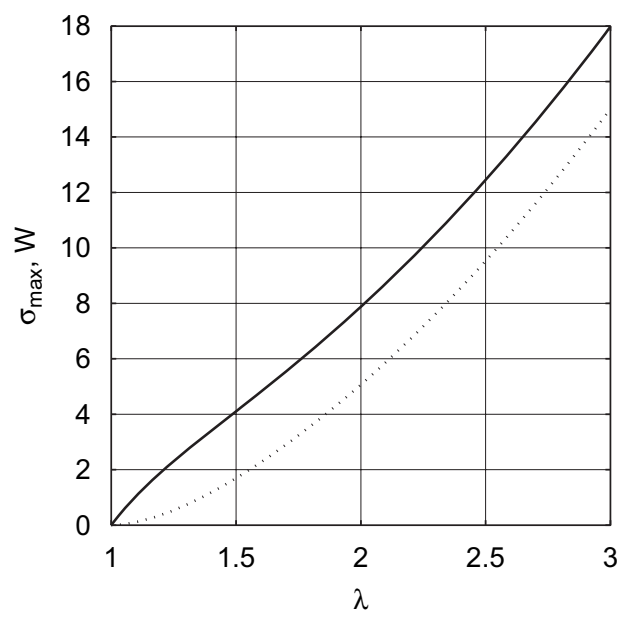

b

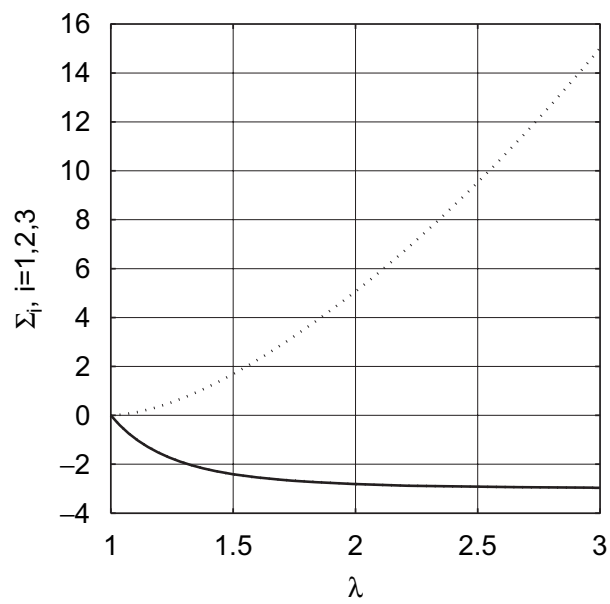

Fig. 6. Equibiaxial tensile results. (a) Maximum principal Cauchy stress (-), strain energy ( $\cdots)$, (b) $\Sigma_{1}=\Sigma_{2}(-), \Sigma_{3}(\cdots)$.

(SBR) (details of compounds are given in the original paper). For uniaxial extension fatigue loading conditions, classical dumb-bell samples are used; for a given stretch level, the experimental end-of-life is the mean value of 7 or 12 samples end-of-life. For equibiaxial extension loading conditions, authors develop a membrane inflation apparatus in which four flat circular sheets are simultaneously inflated; for a given stretch level, the equibiaxial end-of-life is the mean value of 8 tests. It is to note that such bubble inflation technique was widely used to determine stress-strain curves of elastomers or heat-softened polymers (Verron, 1997). Nevertheless, its application to fatigue investigation is not common.

In both uniaxial and equibiaxial cases, the experimental end-of-life is defined as the number of cycles which corresponds to the complete failure of the sample. Recalling that we focus our attention on fatigue crack nucleation, it might be confusing to consider nucleation predictors for the present data. To overcome this difficulty, it is assumed that the propagation of a macroscopic crack until complete failure of the sample is very rapid, in terms of number of cycles, comparing to the nucleation phase, i.e. most of cycles are spent during crack nucleation.

For each fatigue tests, published data are the mean fatigue life in number of cycles $N_{f}$, the maximum stretch level and the strain energy in a cycle. The latter is obtained from integration of the stress-strain curve for uniaxial loading conditions and by simulation for equibiaxial loading conditions. No stress values are given in the paper.

\subsubsection{Prediction}

As loading conditions are proportional, the predictor reduces to $\Sigma^{*}$ given by Eq. (13). In order to calculate classical predictors and $\Sigma^{*}$, constitutive equations of the materials are needed. In this way, for each material strain energy in a cycle vs. stretch level curves for both uniaxial and equibiaxial experiments are simultaneously fitted with a second-order Mooney-Rivlin strain energy function

$$
W=C_{10}\left(I_{1}-3\right)+C_{01}\left(I_{2}-3\right)+C_{20}\left(I_{1}-3\right)^{2} .
$$

Values of the parameters for the four materials are given in Table 1. Similarly to the first example presented above, each predictor can be easily computed. The deformation gradient is given by Eq. (35) with $B=-0.5$ for uniaxial extension and $B=1$ for equibiaxial extension. The maximum stretch is equal to the stretch level $\lambda$ prescribed during experiments and the strain energy is given in the original paper. The maximum principal Cauchy stress is derived from Eq. (15), the relation between the Cauchy and the second Piola-Kirchhoff stress tensor:

$$
\boldsymbol{\sigma}=J^{-1} \mathbf{F S F}^{\mathrm{T}} \text { with } J=1,
$$


Table 1

Parameters for the four materials considered by Roberts and Benzies (1977)

\begin{tabular}{llll}
\hline Material & $C_{10}(\mathrm{MPa})$ & $C_{01}(\mathrm{MPa})$ & $C_{20}(\mathrm{MPa})$ \\
\hline Filled NR & 0.89 & 0.46 & 0 \\
Unfilled NR & 0.44 & 0.056 & 0 \\
Filled SBR & 0 & 1.13 & 0.04 \\
Unfilled SBR & 0.27 & 0.1 & 0.011 \\
\hline
\end{tabular}

and the plane stress condition $\sigma_{33}=0$; then it leads to

$$
\sigma_{\max }=\sigma_{11}=2\left(W_{1}+I_{1} W_{2}\right)\left(\lambda^{2}-\lambda^{-2 B-2}\right)-2 W_{2}\left(\lambda^{4}-\lambda^{-4 B-4}\right),
$$

with

$$
I_{1}=\lambda^{2}+\lambda^{2 B}+\lambda^{-2 B-2}
$$

Finally, recalling Eq. (23), the predictor reduces to

$$
\Sigma^{*}=\left|W-\sigma_{\max }\right|,
$$

where $\sigma_{\max }$ is given by Eq. (41), $W$ is given by Eq. (39), the first strain invariant is given by Eq. (42) and the second strain invariant is

$$
I_{2}=\lambda^{-2}+\lambda^{-2 B}+\lambda^{2 B+2} \text {. }
$$

The performances of the predictors are illustrated in Figs. 7-10 for the maximum stretch, the strain energy, the maximum principal Cauchy stress and the minimum principal configurational stress respectively. In each figure, four graphs are presented, each one corresponds to one of the four materials. In each graph, two Wöhler curves are drawn: one for uniaxial tension data and the other for equibiaxial data. In these curves, one point corresponds to one experiment and its coordinates are the end-of-life (here the logarithm of the number of cycles to failure) and the value of the predictor (the experimental stretch level in Fig. 7, the experimental strain energy in Fig. 8, the maximum principal Cauchy stress given by Eq. (41) in Fig. 9 and our predictor given by Eq. (43) in Fig. 10). As mentioned in the Introduction, the quality of the predictors can be assessed from their ability to unify uniaxial and equibiaxial experimental data, i.e. to superimpose the corresponding Wöhler curves.

For the first three materials, i.e. filled and unfilled NR, and filled SBR, results are similar (see Figs. 7-10(a)-(c)).

- The strain predictor $\lambda_{\max }$ gives relatively good agreement for long fatigue life but important discrepancy for shorter life as shown in Fig. 7(a)-(c). In fact shorter life values correspond to large stretch levels and it is well known that the difference of material response between uniaxial and equibiaxial deformation modes increases as stretch increases (Treloar, 1944).

- For the strain energy predictor $W$, a similar conclusion can be drawn: even if the agreement is roughly better than for the maximum stretch, the discrepancy for large strain is substantial (see Fig. 8(a)-(c)).

- For the stress predictor $\sigma_{\max }$, results are quite better: uniaxial and equibiaxial fatigue curves are superimposed. For filled and unfilled NR, both small and large strain predictions are in good agreement as shown in Fig. 9(a)-(b). For filled SBR, results are not as good as the previous ones but quite better than those predicted with maximum stretch and strain energy (see Fig. 9(c)).

- Finally, Fig. 10(a)-(c) shows the superiority of the proposed predictor with respect to the previously mentioned ones: both small strain (long fatigue life) and large strain (short fatigue life) data are wellpredicted, the best results being obtained for the gum rubber (see Fig. 10(b)).

For the unfilled SBR, results are completely different: only the stretch predictor $\lambda_{\max }$ is able to superimpose the uniaxial and equibiaxial curves. The three other predictors are revealed inefficient (see Figs. 7-10(d)). Until now, we are not capable to satisfactorily interpret this result. Nevertheless, it should be noted that even if the predictor corresponds with microscopic mechanisms in fatigue, particular material behaviours (anisotropy due 
a

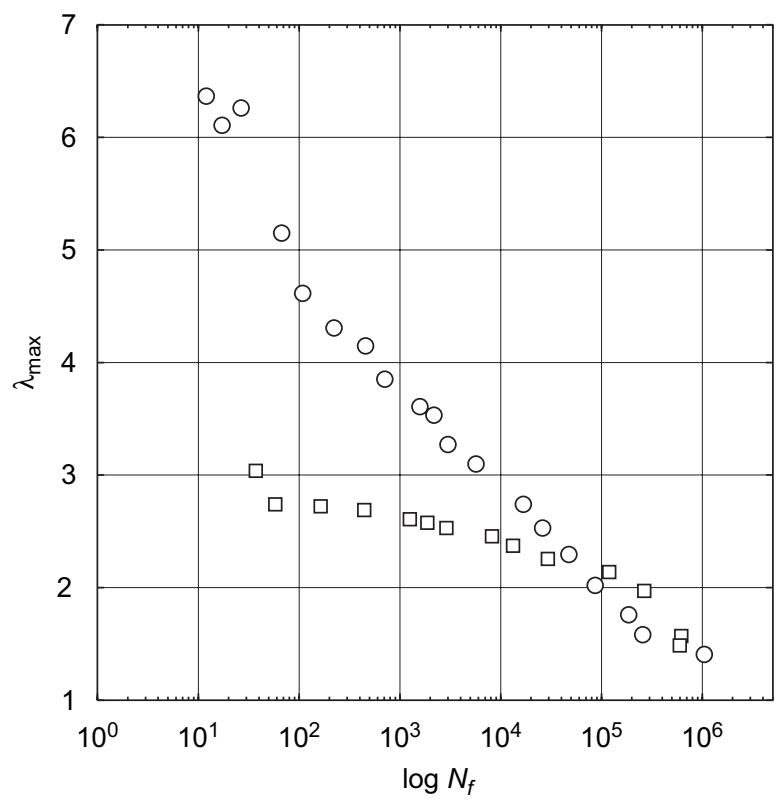

C

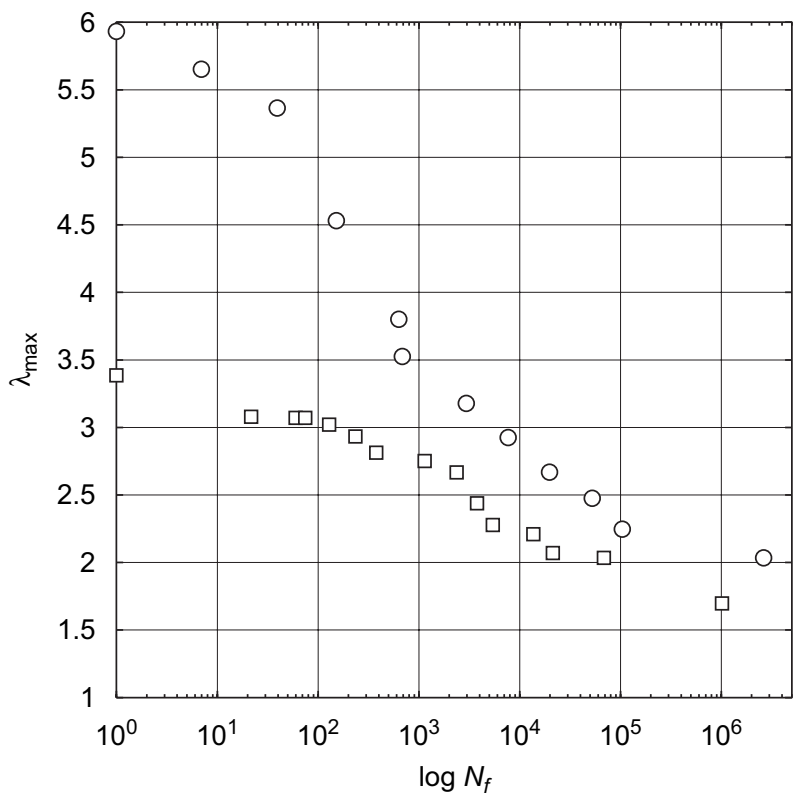

b

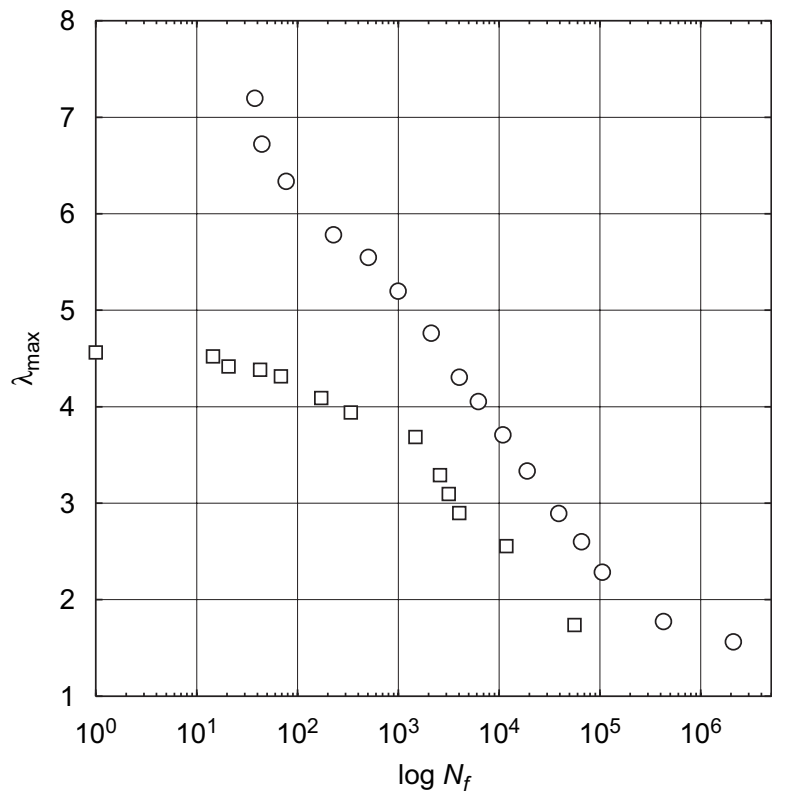

d

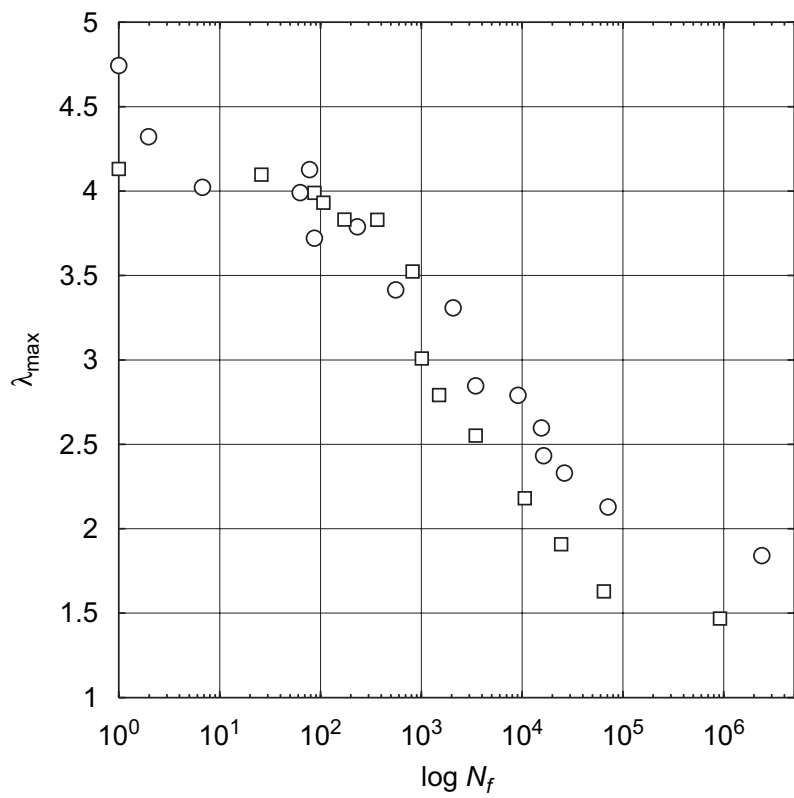

Fig. 7. Comparison of uniaxial $(\bigcirc)$ and equibiaxial $(\square)$ tension fatigue life data of Roberts and Benzies (1977) using the maximum stretch ratio $\lambda_{\max }$. (a) Filled NR, (b) unfilled NR, (c) filled SBR, (d) unfilled SBR.

to finite strain, strain-induced crystallization...) not included in hyperelastic constitutive equations could adversely influence the ability to unify multiaxial experimental results.

\subsection{Multiaxial fatigue prediction}

The aim of this third example is to validate the complete formulation of our new predictor established in Section 3.3 by considering comprehensive multiaxial fatigue experimental results, i.e. both proportional and 
a

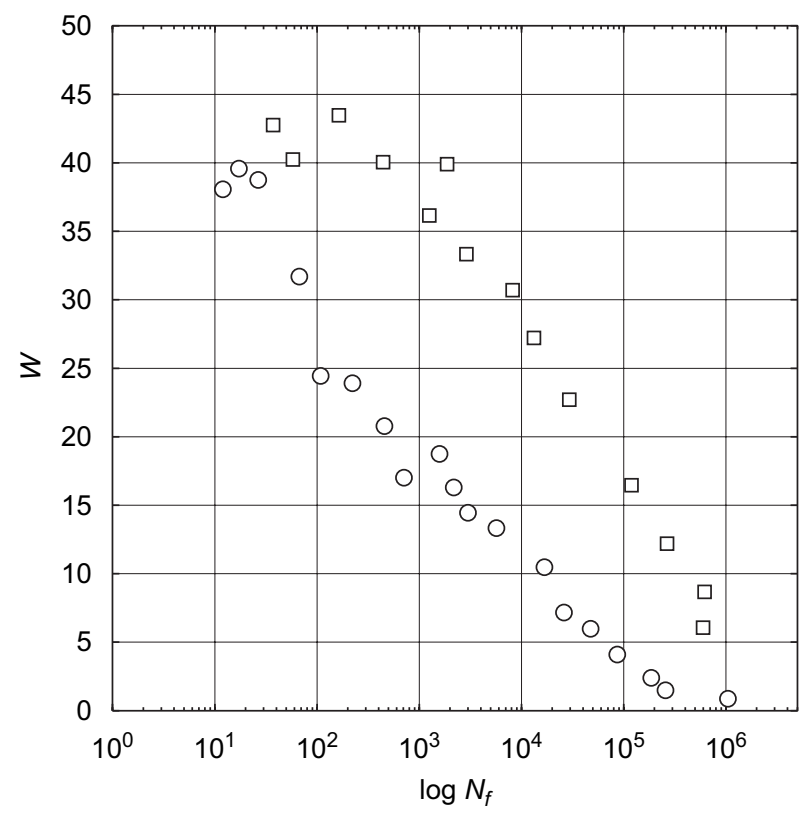

C

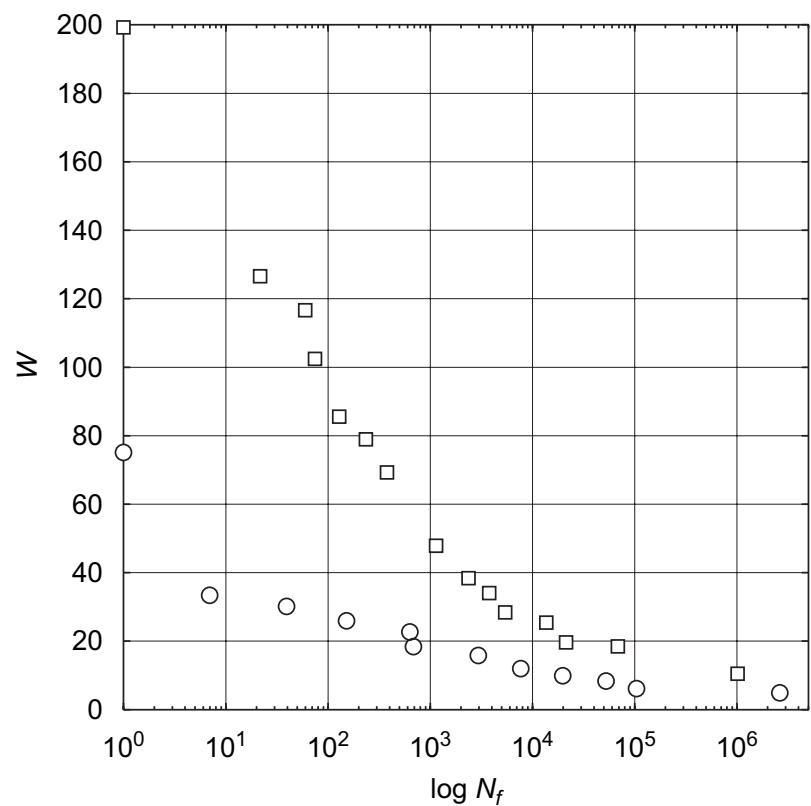

b

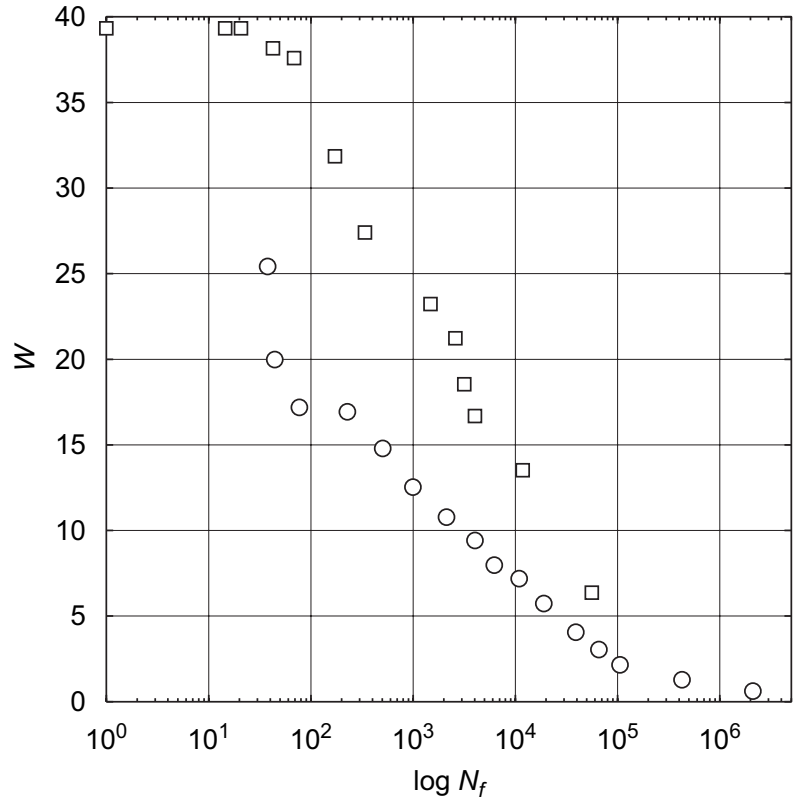

d

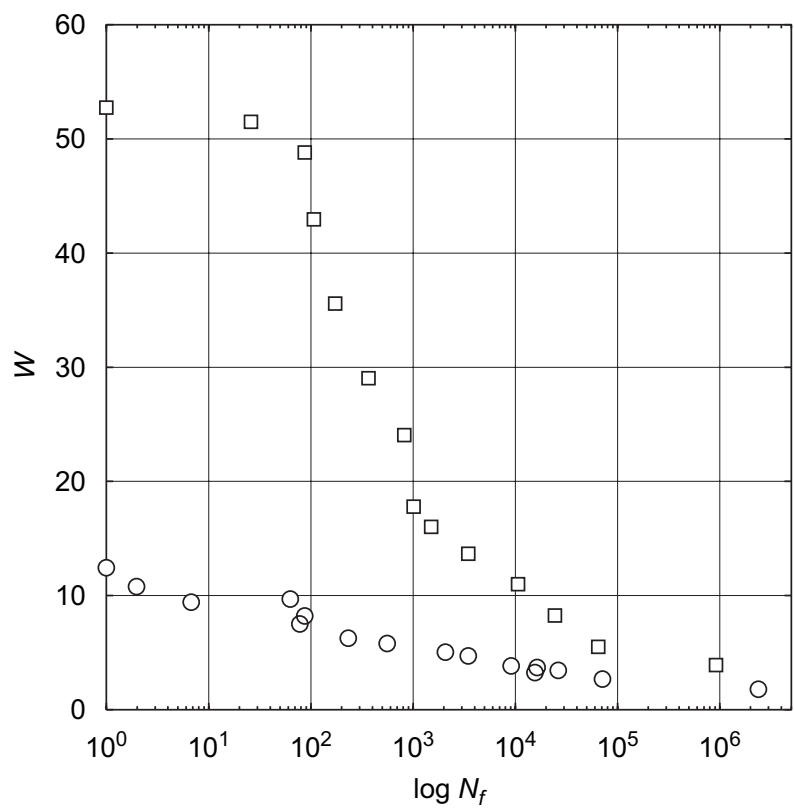

Fig. 8. Comparison of uniaxial $(\bigcirc)$ and equibiaxial $(\square)$ tension fatigue life data of Roberts and Benzies (1977) using the strain energy $W$. (a) Filled NR, (b) unfilled NR, (c) filled SBR, (d) unfilled SBR.

non-proportional multiaxial fatigue data. Such data are rare in the bibliography; here, the outstanding experimental database developed by Mars is retained. The author performed number of uniaxial extension/torsion experiments for complex multiaxial loading conditions: prescribed force or displacement, in- or out-of-phase, fully relaxing and alternate loading conditions were considered. All details concerning samples, loading conditions, measurement methods and results are given in his Ph.D. thesis (Mars, 2001). 
a

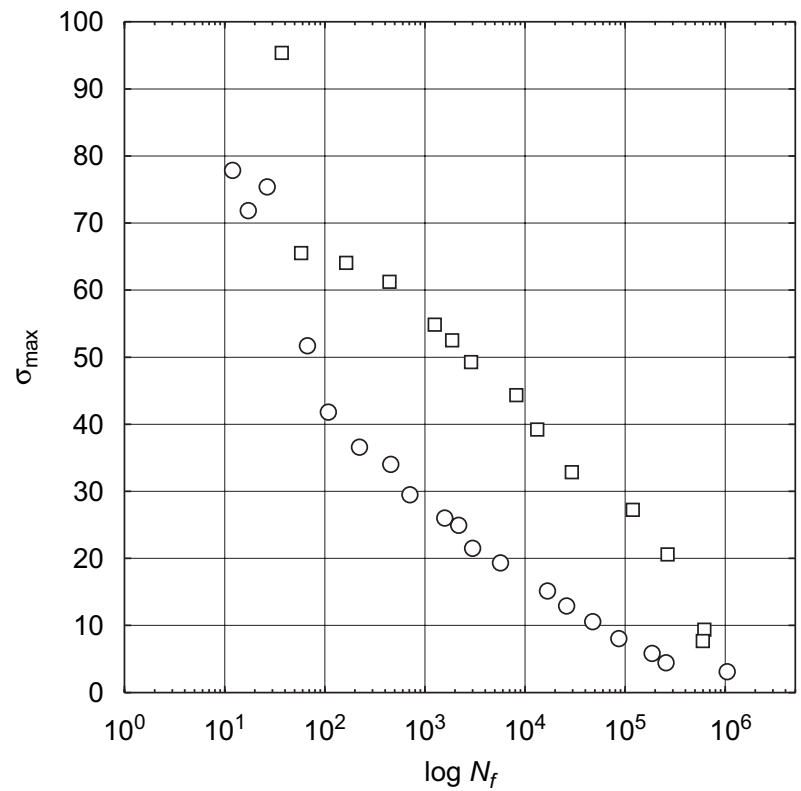

C

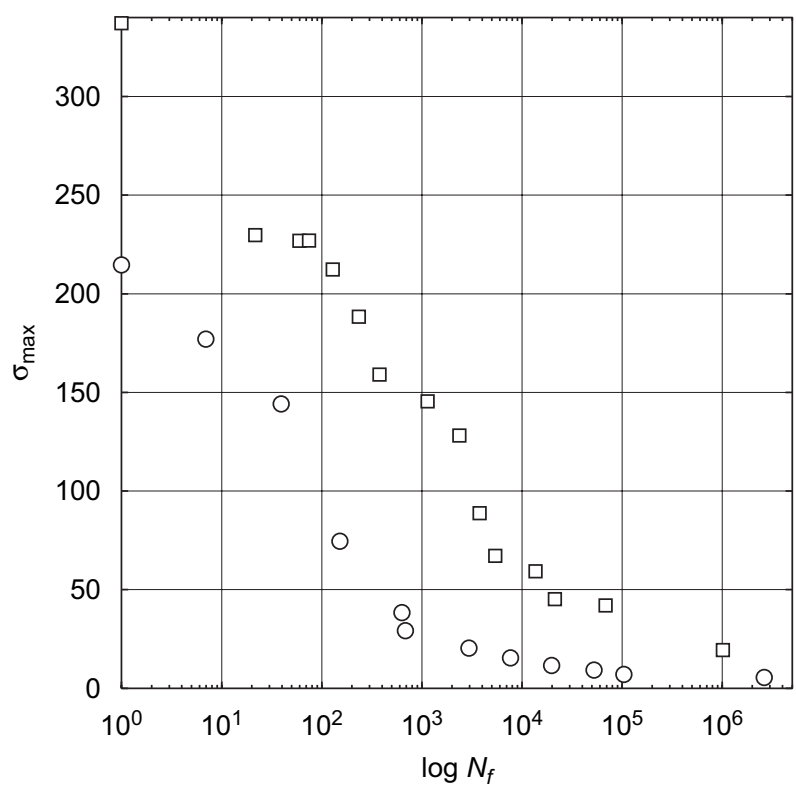

b

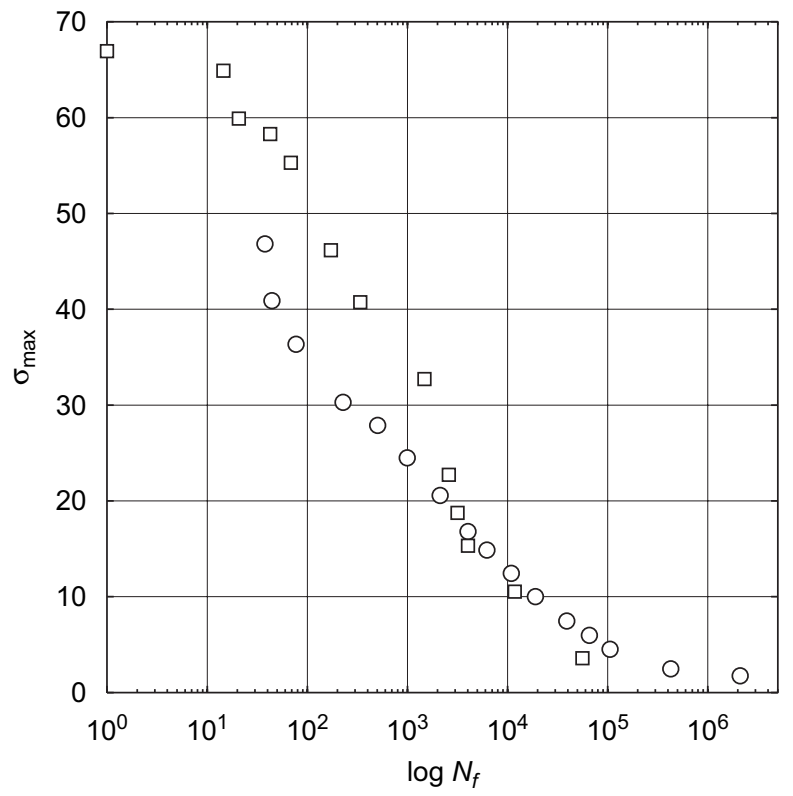

d

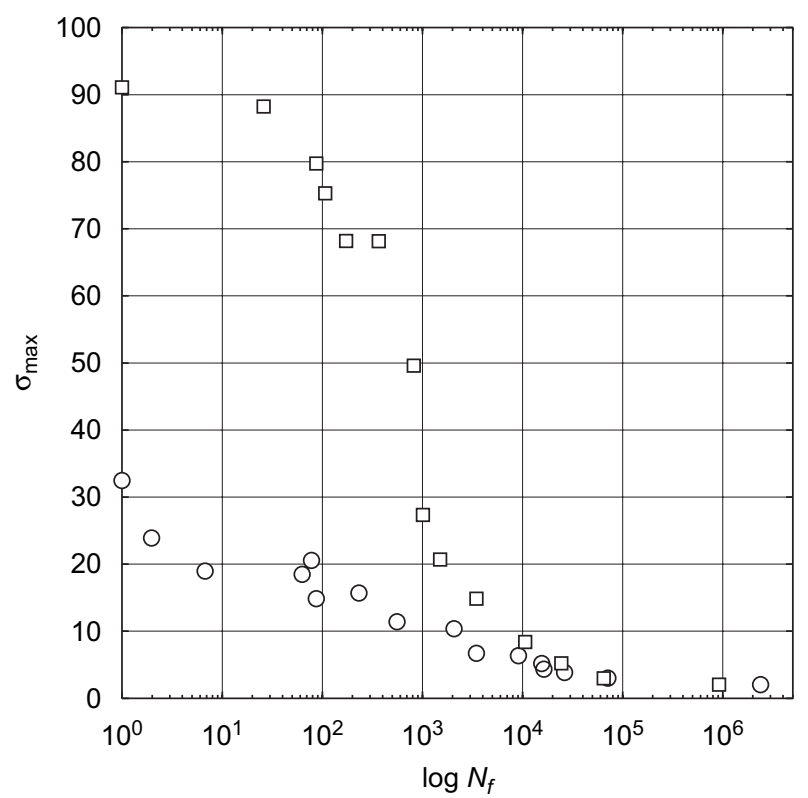

Fig. 9. Comparison of uniaxial $(\bigcirc)$ and equibiaxial $(\square)$ tension fatigue life data of Roberts and Benzies (1977) using the maximum principal Cauchy stress $\sigma_{\max }$. (a) Filled NR, (b) unfilled NR, (c) filled SBR, (d) unfilled SBR.

\subsubsection{Experiments}

In this study, Mars used an industrial carbon black-filled (60 phr) natural rubber and developed an original test specimen. It is a short, hollow cylinder of rubber bonded between two steel mounting rings. Its cross section has a slightly concave surface on the outer diameter and enlarged bonding areas on the top and bottom surfaces. This design was proposed to fulfil, in particular, the following three requirements: to produce a relatively uniform strain field in the sample, to assure that cracks initiate first on the outer surface of the sample and to permit moderate compressive strain without buckling. In the following calculations, the sample geometry is simplified and it is considered as a short thick cylinder which internal radius, external radius and 
a

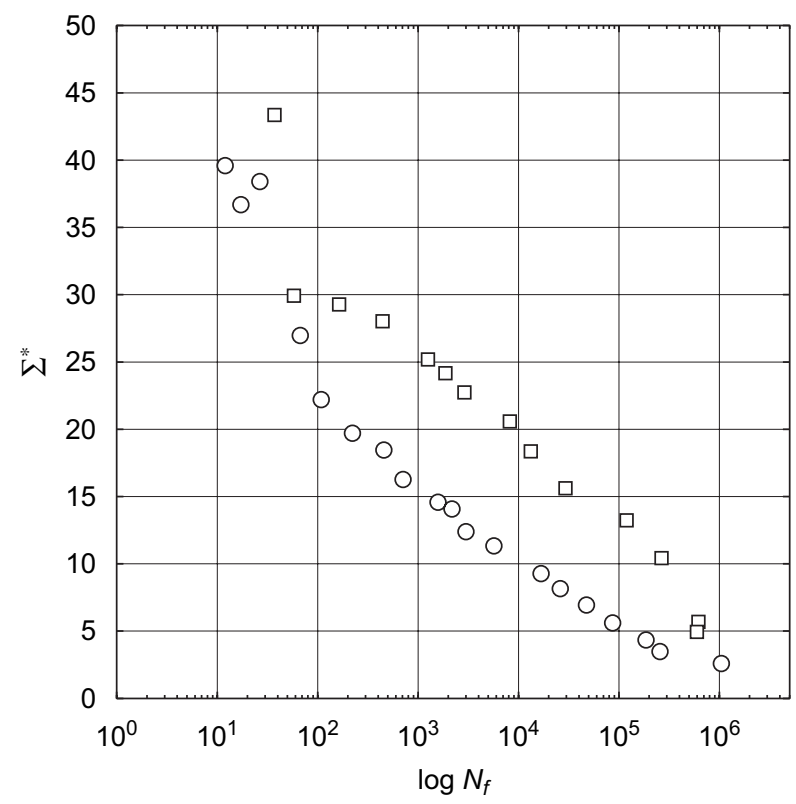

C

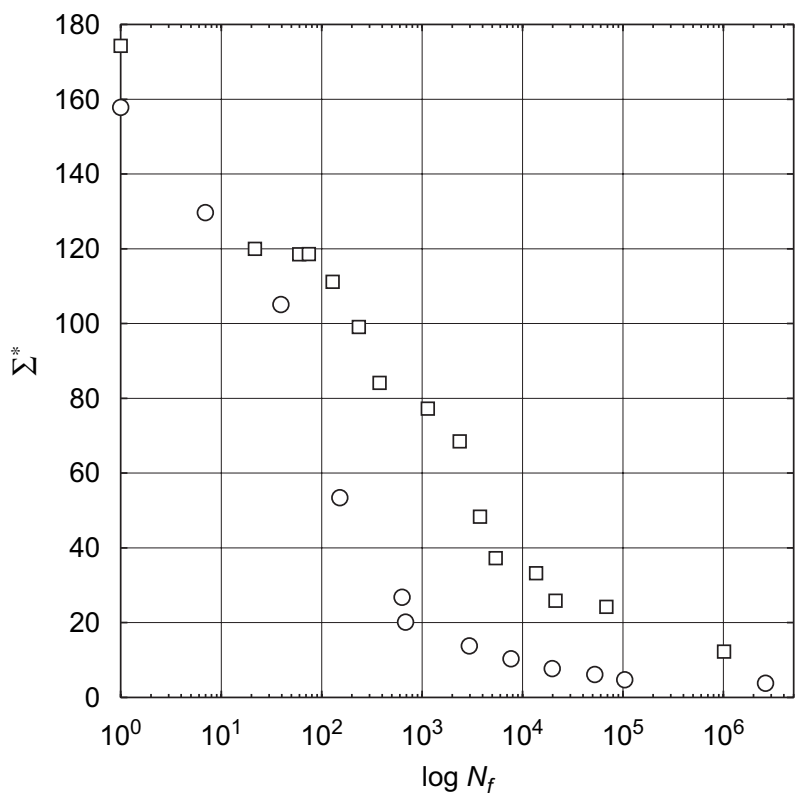

b

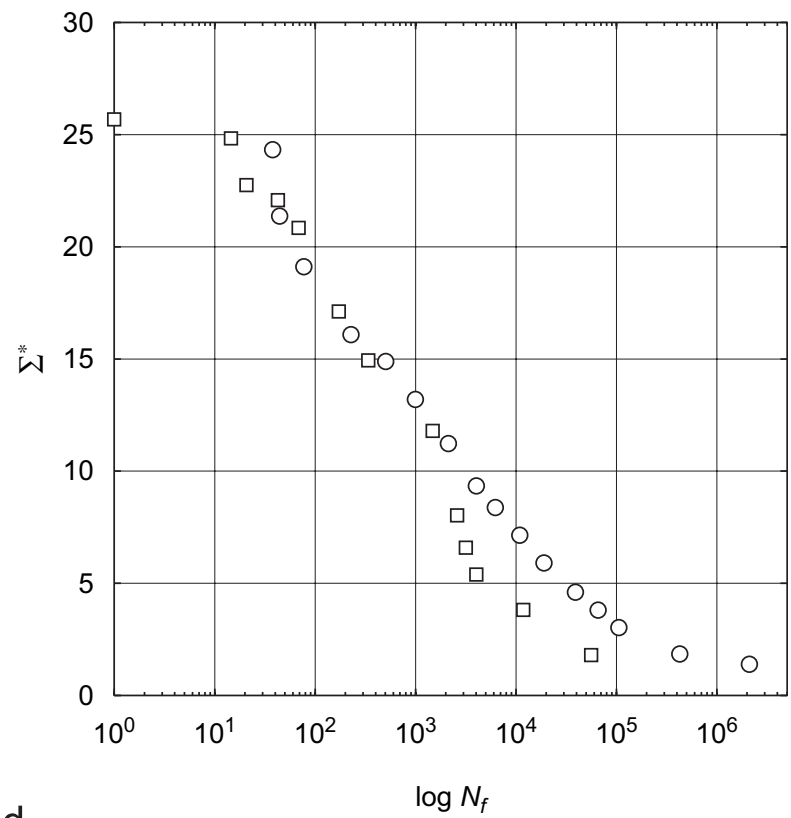

d

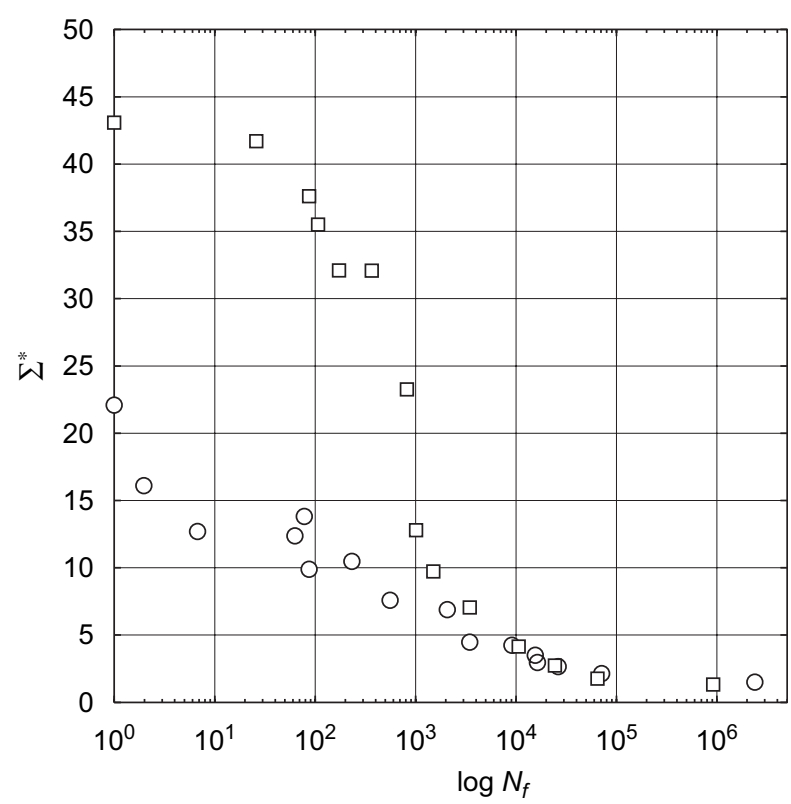

Fig. 10. Comparison of uniaxial $(\bigcirc)$ and equibiaxial $(\square)$ tension fatigue life data of Roberts and Benzies (1977) using the configurational predictor $\Sigma^{*}$. (a) Filled NR, (b) unfilled NR, (c) filled SBR, (d) unfilled SBR.

height are respectively $R_{i}=38.10 \mathrm{~mm}, R_{\mathrm{e}}=43.18 \mathrm{~mm}$ and $h=6.35 \mathrm{~mm}$. According to the author, four main types of cyclic loading conditions are investigated: pure uniaxial extension, pure torsion, proportional uniaxial extension/torsion and non-proportional uniaxial extension/torsion. Displacement field, i.e. axial displacement and twist, or forces, i.e. load and torque, can be prescribed; in the present work only prescribed displacement loading conditions are retained. Thus, loading conditions can be written as

$$
\lambda(t)=\lambda_{\mathrm{m}}+\lambda_{\mathrm{a}} \sin (\omega t) \quad \text { and } \quad \tau(t)=\tau_{\mathrm{m}}+\tau_{\mathrm{a}} \sin (\omega t+\phi),
$$


where $\lambda$ is the stretch ratio in the axial direction and $\tau$ stands for the twist per unit length. For a given experimental test, six parameters are prescribed: the mean stretch ratio $\lambda_{\mathrm{m}}$ and its amplitude $\lambda_{\mathrm{a}}$, the mean twist $\tau_{\mathrm{m}}$ and its amplitude $\tau_{\mathrm{a}}$, the signal frequency $2 \pi / \omega$ which varies between 0.5 and $6.0 \mathrm{~Hz}$, and finally the phase angle $\phi$ between extension and twist. Loading conditions are said proportional when $\phi=0$. Moreover, classical extension and twist loading ratios are, respectively, defined by $R_{\lambda}=\left(\lambda_{\min }-1\right) /\left(\lambda_{\max }-1\right)$ and $R_{\tau}=\tau_{\min } / \tau_{\max }$. The ten different types of loading conditions (from A to I, and L) considered here are sketched in Fig. 11 and the corresponding loading parameters are given in Table 2; designations are those proposed by Mars. In Mars (2001), two independent end-of-life criteria for crack nucleation were applied. The first criterion is based on load and torque drop. More precisely, the load or torque drop which corresponds to endof-life is defined as the number of cycles $N_{f}$ at which loading (load or torque) amplitude was $15 \%$ less than its stabilized value (defined by the loading value at 128 cycles in this study). The second criterion is based on photographic observation of cracks growth on sample surface and on the use of a crack growth model. In this case, end-of-life corresponds with the occurrence of a $0.2-0.8 \mathrm{~mm}$ long crack on sample surface. Indeed, both failure criteria resulted in similar fatigue life to within a factor of about 2 . Here we only adopt the value of $N_{f}$ given by the first criterion. Finally, for prescribed displacement tests, the retained experimental data are the number of cycles at end-of-life $N_{f}$ and the crack angle in the undeformed configuration.

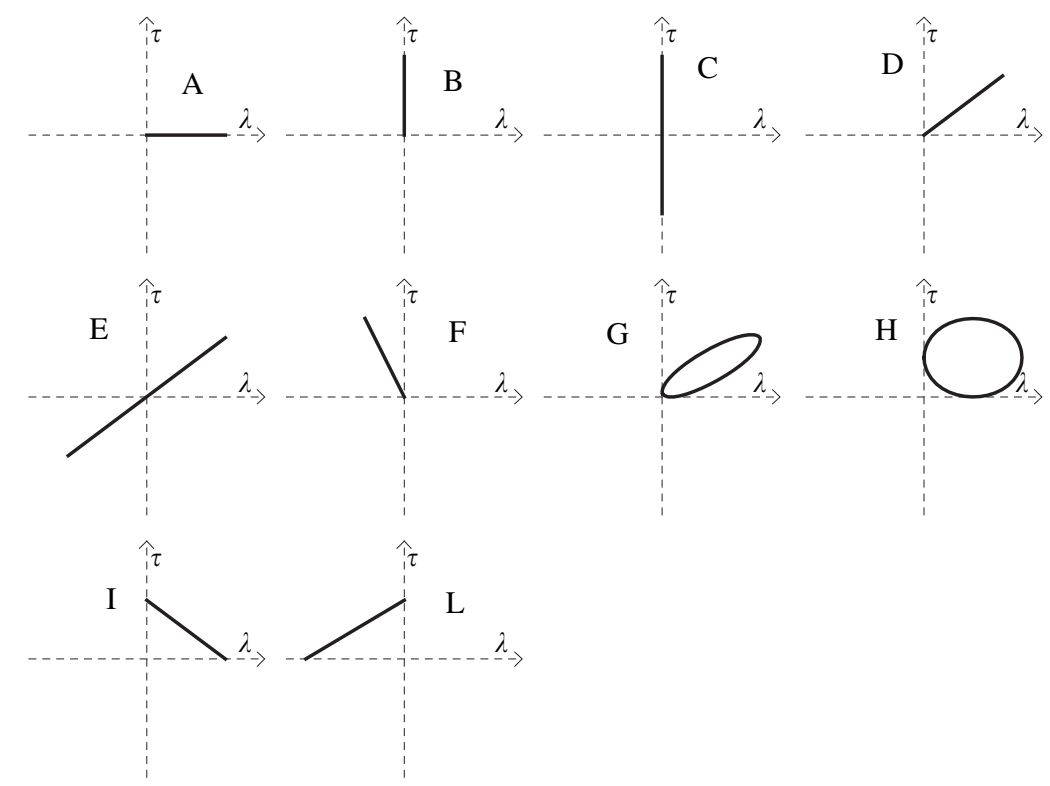

Fig. 11. Simultaneous uniaxial extension and torsion of a rubber cylindrical tube: different types of loading conditions as proposed by Mars (2001).

Table 2

Loading parameters for Mars experiments

\begin{tabular}{lllllllllll}
\hline Data set & A & B & C & D & E & F & G & H & I & L \\
\hline Number of exp. & $13(11)$ & $12(10)$ & $9(8)$ & 16 & 5 & 3 & 3 & 5 & $4(3)$ & $5(1)$ \\
Extension ratio $R_{\lambda}$ & 0 & - & - & 0 & -1 & $-\infty$ & 0 & 0 & 0 \\
Twist ratio $R_{\tau}$ & - & 0 & -1 & 0 & -1 & 0 & 0 & 0 & 0 \\
Phase angle $\phi$ & 0 & 0 & 0 & 0 & 0 & $-\pi$ & $\pi / 4$ & $\pi / 2$ & $-\pi$ & 0 \\
\hline
\end{tabular}

For the number of experiments, the number in brackets stands for the number of experiments for which it was possible to measure the crack angle. 


\subsubsection{Predictions}

For each loading conditions, i.e. for each experiment, the predictor $\Sigma^{*}$ is determined by solving the problem of simultaneous uniaxial extension and torsion of a hyperelastic cylinder. The analytical solution of this problem can be found for example in Green and Adkins (1960). The computation of the configurational stress tensor in this case is given in the Appendix. Following Mars, the material is supposed to obey the neoHookean model with $C=1.5$. Once the configurational stress increment $\mathrm{d} \boldsymbol{\Sigma}$ being determined as a function of $\lambda$ and $\tau$ (see Eqs. (53)-(55)), the value of $\Sigma^{*}$ and the crack angle, i.e. more precisely the normal vector to the crack plane $\mathbf{V}^{*}$, are calculated by using Eqs. (13) and (26)-(31).

The data are now used to validate our approach. In this way, it has been first verified that the predictor is maximized at the external radius $R_{\mathrm{e}}$ of the cylinder, thus, as predicted by Mars during sample design, macroscopic fatigue cracks initiate first in the outer surface of the specimen for every loading cases. In order to simplify the discussion, three different abilities of the new predictor are successively investigated by considering three different sets of experimental data given in Fig. 11 and Table 2. First, the ability to manage multiaxial loading conditions is studied by considering $R=0$ proportional loading conditions, i.e. data $\mathrm{A}$, B, $\mathrm{D}$ and $\mathrm{F}$. Second, the ability to accumulate the damage is assessed by comparing $R=0$ and -1 proportional loading conditions, i.e. data B, C, D and E. Third, the ability to predict non-proportional loading conditions, i.e. $\phi \neq 0$ is examined by comparing proportional and non-proportional loading conditions, i.e. data $D, G, H$, I , F and L. In these three cases, two graphs are drawn. The first graph (left-hand side graph of the figures) presents the Wöhler curve, i.e. the value of the predictor for given loading conditions $\Sigma^{*}$ vs. the corresponding experimental end-of-life $\log N_{f}$. Similarly to the previous example, the efficiency of the predictor is assessed from its ability to unify the data. The second graph (right-hand side graph in the subsequent figures) presents the predicted crack angle vs. the measured crack angle; the diagonal line represents the case where the measured crack orientation is exactly predicted by the theory.

Results dedicated to the ability to predict multiaxial proportional fatigue life are presented in Fig. 12. The ability of the present approach to unify $R=0$ and -1 is illustrated in Fig. 13. Finally, the relevance of nonproportional predictions is demonstrated in Fig. 14. In the left-hand side graph, one experimental point of the data set L was not considered because it corresponds to $N_{f}=76$ cycles which is inferior to the number of cycles for which the material response is assumed stabilized (128 cycles). Moreover, in the right-hand side graph, two experimental points of the set I were not drawn in order to keep readable axis scales; the measured angles are $43^{\circ}$ and $50^{\circ}$ and the corresponding predictions are $-10^{\circ}$ and $-23^{\circ}$. The three figures can be commented simultaneously because they lead to the same conclusion. Left-hand side graphs demonstrate the ability of the present approach to unify different multiaxial fatigue data in one Wöhler curve. Thus, the three abilities proposed above are satisfied. Indeed, all data collected in a unique graph would be also superimposed: curves shape and axis scales are similar in Figs. 12-14. In this case, all data could be fitted by a straight line. Concerning the prediction of crack angles shown in the right-hand side graphs of Figs. 12-14, predictions are also in good quite agreement with experimental data for all loading conditions. The only exception is for alternate $(R=-1)$ torsion experiments (set C) in Fig. 13 and non-proportional $\phi=\pi$ uniaxial extension/ torsion experiments (set I) as noted above. As shown by photographic data in Mars (2001, p. 231 and 278), there are some uncertainties in the measure crack angles. It can be explained by the fact that a macroscopic crack consists of a large number of microscopic cracks with different orientation (see Le Cam, 2005, p. 79).

\section{Summary and conclusions}

We have derived a new predictor $\Sigma^{*}$ for multiaxial fatigue crack nucleation in rubber materials. Its formulation is motivated by the observation of the microscopic damage induced by fatigue: first, the nucleation of a macroscopic fatigue crack is the consequence of the propagation of pre-existing flaws in the material; and second, most of cycles experienced by rubber specimen until complete failure are spent during flaws growth. Following these observations, the theoretical derivation of our predictor is founded on the framework of Configurational Mechanics, described as "a tool to capture singularities" by Maugin (1995). In order to calculate the energy release rate of microscopic flaws, the peculiar properties of the configurational stress tensor are considered. Knowing that most of these flaws are micro-voids which grow under traction, the predictor is given by the smallest negative eigenvalue of the damage part of this stress tensor. The method to 

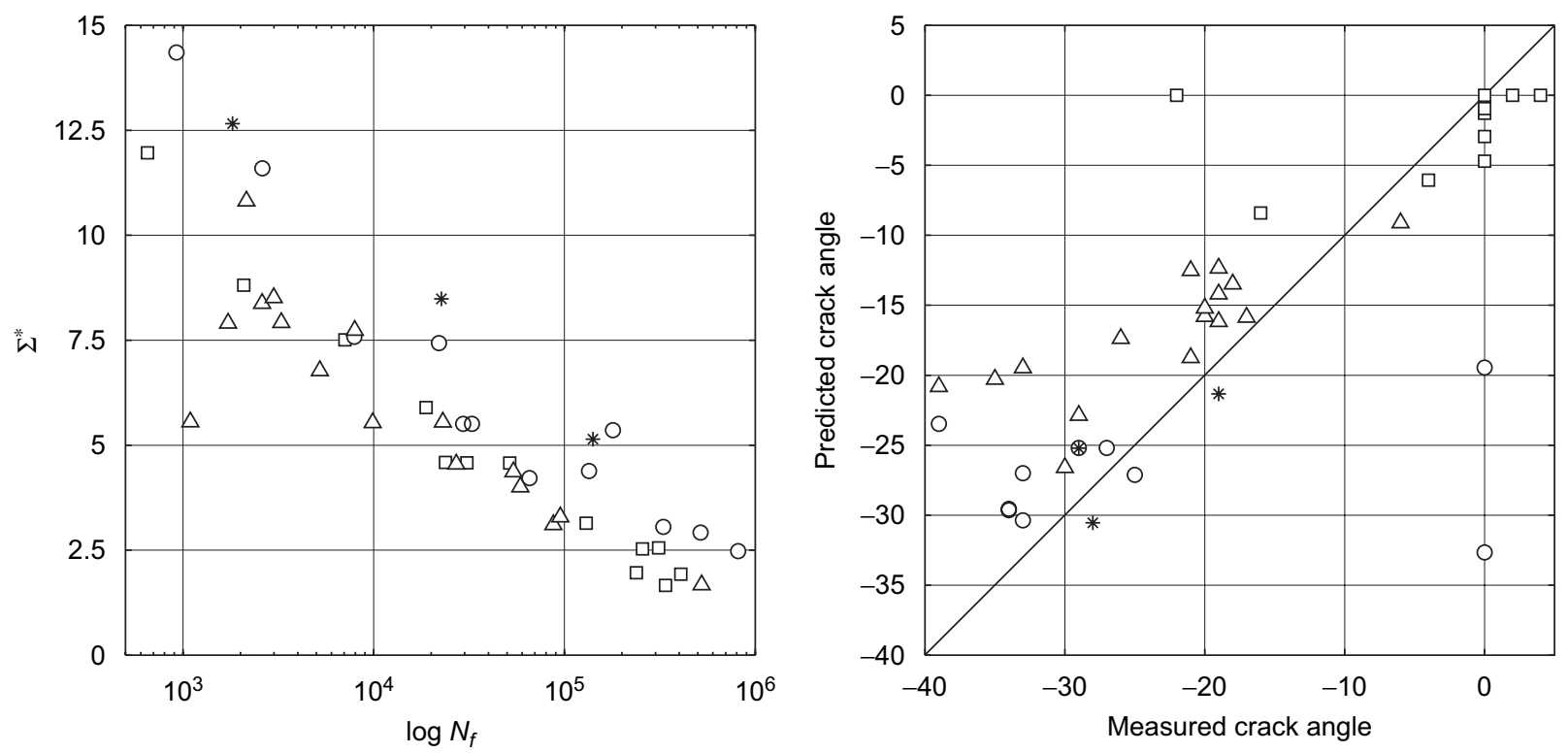

Fig. 12. Comparison of $R=0$ proportional loading conditions: ( $\square$ ) uniaxial extension test (A), $(\bigcirc)$ torsion test (B), $(\triangle)$ uniaxial extension/torsion test (D), (*) uniaxial compression/torsion test (F).
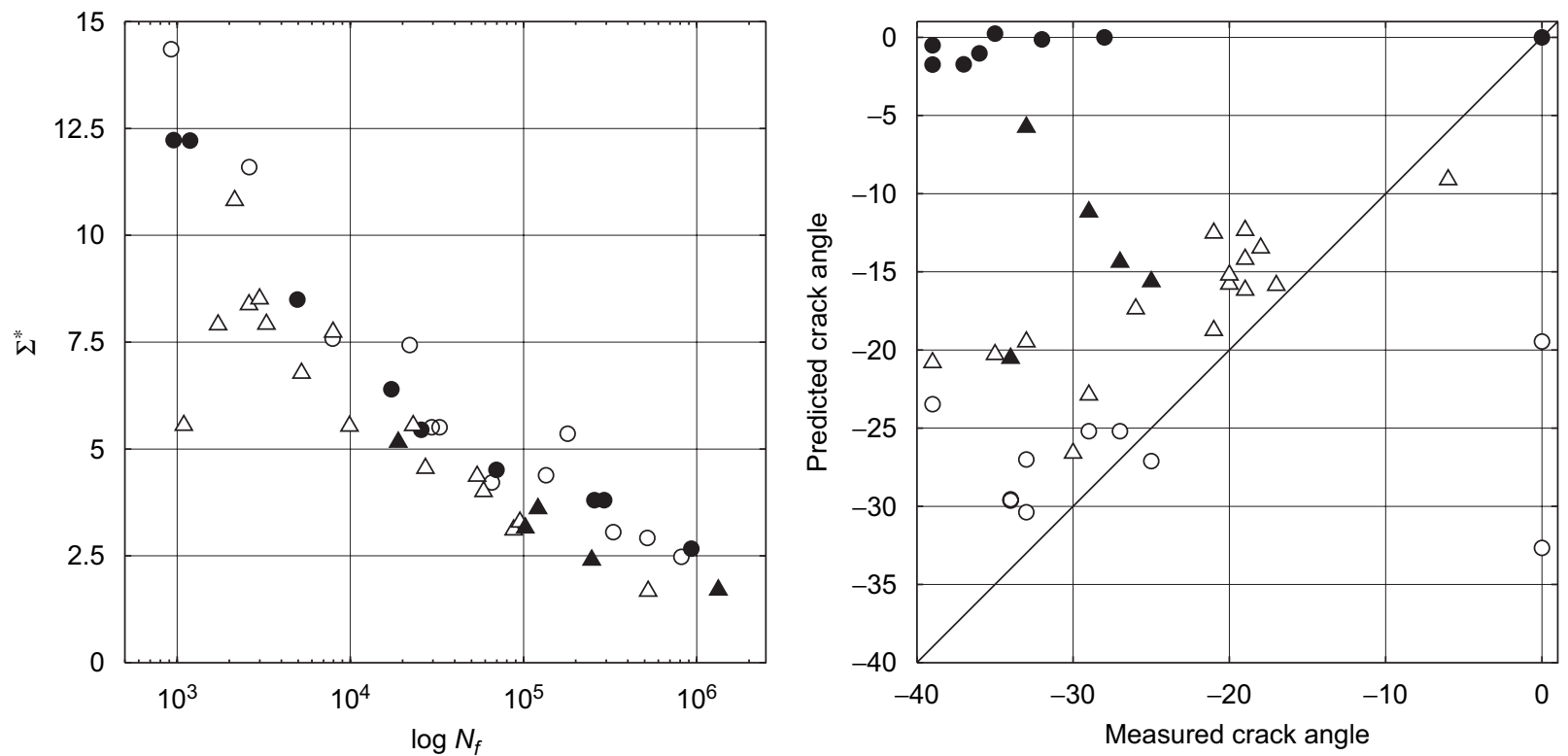

Fig. 13. Comparison of $R=0$ and -1 proportional loading conditions: $(\bigcirc) R=0$ torsion test $(\mathrm{B}),(\bullet) R=-1$ torsion test $(\mathrm{C}),(\triangle)$ $R=0$ uniaxial extension/torsion test $(\mathrm{D}),(\boldsymbol{\Delta}) R=-1$ uniaxial extension/torsion test $(\mathrm{E})$.

accumulate the damage part of the configurational stress tensor for non-proportional multiaxial fatigue has also been proposed. It should be noted that $\Sigma^{*}$ is written in terms of continuum mechanics quantities (strain, stress, energy). Thus, it can be easily computed for ideal rubber parts (without considering defects) during product development process in order to estimate the spatial distribution of fatigue life. The efficiency of our proposal is assessed by considering experimental data in the literature. It is demonstrated that the new predictor, which rationalizes the previously published predictors, is capable to unify multiaxial fatigue data. 

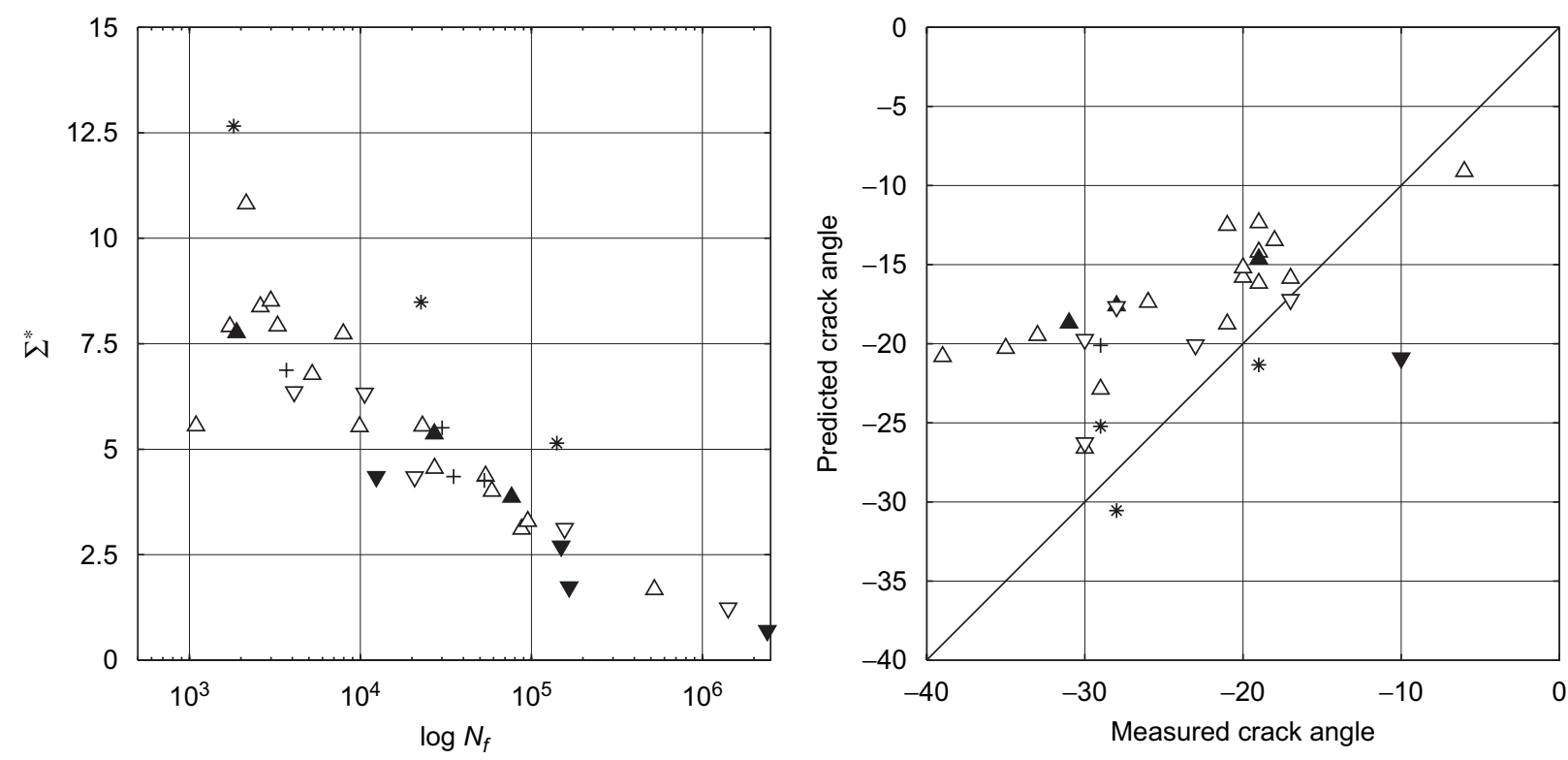

Fig. 14. Comparison of proportional and non-proportional loading conditions: $(\triangle)$ proportional uniaxial extension/torsion test $(\mathrm{D}),(\boldsymbol{\Delta})$ non-proportional $\phi=\pi / 4$ uniaxial extension/torsion test $(\mathrm{G}),(\nabla)$ non-proportional $\phi=\pi / 2$ uniaxial extension/torsion test $(\mathrm{H}),(\boldsymbol{\nabla})$ nonproportional $\phi=-\pi$ uniaxial extension/torsion test (I), (*) proportional uniaxial compression/torsion test $(\mathrm{F}),(+)$ non-proportional $\phi=\pi$ uniaxial compression/torsion test (L).

Some important issues are, however, left unresolved. First, as most of the authors, we have considered that flaws in rubber grow only due to material traction. A thorough study of this assumption would be necessary to precisely determine which part of the configurational stress tensor induces fatigue damage. Second, the relevant mechanical quantity that should be experimentally measured to develop a complete fatigue criterion for given materials should be determined, and the corresponding experimental methods should be defined. Finally, until now, the application of our theory is limited to simple problems; the numerical implementation of the predictor, and more precisely of its accumulation during a cycle, is currently under investigation.

\section{Appendix A. Uniaxial extension/torsion of a hyperelastic tube}

This appendix summarizes the derivation of the governing equations for the simultaneous uniaxial extension and torsion of a cylindrical hyperelastic tube. Moreover, the configurational stress tensor and its increment are derived in order to compute the multiaxial predictor $\Sigma^{*}$.

Let $(R, \Theta, Z)$ and $\left(\mathbf{e}_{R}, \mathbf{e}_{\Theta}, \mathbf{e}_{Z}\right)$ be the coordinates and the cylindrical unit vectors in the undeformed configuration. Let $(r, \theta, z)$ and $\left(\mathbf{e}_{r}, \mathbf{e}_{\theta}, \mathbf{e}_{z}\right)$ denote their counterparts in the deformed configuration. The deformation of simultaneous uniaxial extension and torsion of a circular tube is given by

$$
r=\frac{R}{\sqrt{\lambda}} ; \quad \theta=\Theta+\lambda \tau Z ; \quad z=\lambda Z,
$$

where $\lambda$ is the stretch ratio and $\tau$ is the twist per unit length. The corresponding deformation gradient is

$$
\mathbf{F}=\frac{1}{\sqrt{\lambda}}\left(\mathbf{e}_{r} \otimes \mathbf{e}_{R}+\mathbf{e}_{\theta} \otimes \mathbf{e}_{\Theta}\right)+R \sqrt{\lambda} \tau \mathbf{e}_{\theta} \otimes \mathbf{e}_{Z}+\lambda \mathbf{e}_{z} \otimes \mathbf{e}_{Z}
$$

and left and right Cauchy-Green strain tensors can be expressed as

$$
\mathbf{B}=\frac{1}{\lambda} \mathbf{e}_{r} \otimes \mathbf{e}_{r}+\left(\frac{1}{\lambda}+\lambda^{2} \tau^{2} r^{2}\right) \mathbf{e}_{\theta} \otimes \mathbf{e}_{\theta}+\lambda^{2} \tau r\left(\mathbf{e}_{\theta} \otimes \mathbf{e}_{z}+\mathbf{e}_{z} \otimes \mathbf{e}_{\theta}\right)+\lambda^{2} \mathbf{e}_{z} \otimes \mathbf{e}_{z}
$$


and

$$
\mathbf{C}=\frac{1}{\lambda}\left(\mathbf{e}_{R} \otimes \mathbf{e}_{R}+\mathbf{e}_{\Theta} \otimes \mathbf{e}_{\Theta}\right)+\tau R\left(\mathbf{e}_{\Theta} \otimes \mathbf{e}_{Z}+\mathbf{e}_{Z} \otimes \mathbf{e}_{\Theta}\right)+\left(\lambda^{2}+\lambda \tau^{2} R^{2}\right) \mathbf{e}_{Z} \otimes \mathbf{e}_{Z}
$$

Using the constitutive equation (15) with $\partial W / \partial I_{1}=C$ and $\partial W / \partial I_{2}=0$, the relation between Cauchy and second Piola-Kirchhoff stress tensor Eq. (40), equilibrium equations and the boundary condition which imposes that the external lateral surface $\left(R=R_{\mathrm{e}}\right)$ is traction free, the pressure $p$ is derived

$$
p(R)=2 C \frac{1}{\lambda}+C \lambda \tau^{2}\left(R_{\mathrm{e}}^{2}-R^{2}\right)
$$

and the Cauchy stress can be expressed as a function of the undeformed radius:

$$
\begin{aligned}
\boldsymbol{\sigma}(R)= & C \lambda \tau^{2}\left(R^{2}-R_{\mathrm{e}}^{2}\right) \mathbf{e}_{r} \otimes \mathbf{e}_{r}+C \lambda \tau^{2}\left(3 R^{2}-R_{\mathrm{e}}^{2}\right) \mathbf{e}_{\theta} \otimes \mathbf{e}_{\theta}+2 C \lambda^{\frac{3}{2}} \tau R \\
& \times\left(\mathbf{e}_{\theta} \otimes \mathbf{e}_{z}+\mathbf{e}_{z} \otimes \mathbf{e}_{\theta}\right)+\left[2 C\left(\lambda^{2}-\frac{1}{\lambda}\right)+C \lambda \tau^{2}\left(R^{2}-R_{\mathrm{e}}^{2}\right)\right] \mathbf{e}_{z} \otimes \mathbf{e}_{z} .
\end{aligned}
$$

Thus, using Eqs. (49)-(51) and the relationship $\mathbf{S}=J \mathbf{F}^{-1} \boldsymbol{\sigma} \mathbf{F}^{-\mathrm{T}}$, the configurational stress tensor reduces to

$$
\begin{aligned}
\boldsymbol{\Sigma}= & C\left(\lambda^{2}+\frac{2}{\lambda}+\lambda \tau^{2} R_{\mathrm{e}}^{2}-3\right)\left(\mathbf{e}_{R} \otimes \mathbf{e}_{R}+\mathbf{e}_{\Theta} \otimes \mathbf{e}_{\Theta}\right)-2 C \tau R\left(\mathbf{e}_{\Theta} \otimes \mathbf{e}_{Z}\right. \\
& \left.+\mathbf{e}_{Z} \otimes \mathbf{e}_{\Theta}\right)+C\left[-\lambda^{2}+\frac{4}{\lambda}+\lambda \tau^{2}\left(R_{\mathrm{e}}^{2}-R^{2}\right)-3\right] \mathbf{e}_{Z} \otimes \mathbf{e}_{Z} .
\end{aligned}
$$

Finally, the integration of the configurational stress tensor over a fatigue cycle necessitates the determination of its increment

$$
\mathbf{d} \mathbf{\Sigma}=\left(\frac{\mathbf{d} \boldsymbol{\Sigma}}{\mathbf{d} \lambda} \dot{\lambda}+\frac{\mathbf{d} \boldsymbol{\Sigma}}{\mathbf{d} \tau} \dot{\tau}\right) \mathrm{d} t
$$

where

$$
\frac{\mathbf{d} \mathbf{\Sigma}}{\mathbf{d} \lambda}=C\left(2 \lambda-\frac{2}{\lambda^{2}}+\tau^{2} R_{\mathrm{e}}^{2}\right)\left(\mathbf{e}_{R} \otimes \mathbf{e}_{R}+\mathbf{e}_{\Theta} \otimes \mathbf{e}_{\Theta}\right)+C\left[-2 \lambda-\frac{4}{\lambda^{2}}+\tau^{2}\left(R_{\mathrm{e}}^{2}-2 R^{2}\right)\right] \mathbf{e}_{Z} \otimes \mathbf{e}_{Z}
$$

and

$$
\frac{\mathbf{d} \boldsymbol{\Sigma}}{\mathbf{d} \tau}=2 C \lambda \tau R_{\mathrm{e}}^{2}\left(\mathbf{e}_{R} \otimes \mathbf{e}_{R}+\mathbf{e}_{\Theta} \otimes \mathbf{e}_{\Theta}\right)-2 C R\left(\mathbf{e}_{\Theta} \otimes \mathbf{e}_{Z}+\mathbf{e}_{Z} \otimes \mathbf{e}_{\Theta}\right)+2 C \lambda \tau\left(R_{\mathrm{e}}^{2}-2 R^{2}\right) \mathbf{e}_{Z} \otimes \mathbf{e}_{Z}
$$

\section{References}

Abraham, F., Alshuth, T., Jerrams, S., 2005. The effect of minimum stress and stress amplitude on the fatigue life of non strain crystallising elastomers. Mater. Design 26, 239-245.

André, N., Cailletaud, G., Piques, R., 1999. Haigh diagram for fatigue crack initiation prediction of natural rubber components. Kautsch. Gummi Kunstst. 52, 120-123.

Andriyana, A., 2006. Définition d'une nouvelle grandeur prédictive pour la durée de vie en fatigue des matériaux élastomères. Ph.D. Thesis, Ecole Centrale de Nantes.

Andriyana, A., Verron, E., 2007. Prediction of fatigue life improvement in natural rubber using configurational stress. Int. J. Solids Struct. 44, 2079-2092.

ASTM, 1994. Standard test method for rubber property - extension cyclic fatigue. ASTM D 4482-4485.

Baker, M., Ericksen, J.L., 1954. Inequalities restricting the form of the stress-deformation relations for isotropic elastic solids and ReinerRivlin fluids. J. Wash. Acad. Sci. 44, 33-35.

Bathias, C., Houel, P., Berete, Y.N., Legorju, K., 1998. Damage characterization of elastomeric composites using X-Ray attenuation. In: Reifsnider, K.L., Dillard, D.A., Cardon, A.H. (Eds.), Fatigue and Fracture: Progress in Durability Analysis of Composite Systems. Balkema, Rotterdam, pp. 103-110.

Budiansky, B., Rice, J.R., 1973. Conservation laws and energy-release rates. J. Appl. Mech. 40, 201-203.

Cadwell, S.M., Merrill, R.A., Sloman, C.M., Yost, F.L., 1940. Dynamic fatigue life of rubber. Rubber Chem. Technol. 13, 304-315.

Chadwick, P., 1975. Applications of an energy-momentum tensor in non-linear elastostatics. J. Elastricity 5, 249-258. 
Denzer, R., Barth, F.J., Steinmann, P., 2003. Studies in elastic fracture mechanics based on the material force method. Int. J. Numer. Methods Eng. 58, 1817-1835.

Epstein, M., Maugin, G.A., 1990. The energy-momentum tensor and material uniformity in finite elasticity. Acta Mech. 83, 127-133.

Eshelby, J.D., 1951. The force on an elastic singularity. Philos. Trans. R. Soc. London, Ser. A 244, 87-112.

Eshelby, J.D., 1975. The elastic energy-momentum tensor. J. Elasticity 5, 321-335.

Fielding, J.H., 1943. Flex life and crystallization of synthetic rubber. Ind. Eng. Chem. 35, 1259-1261.

Gehman, S.D., Clifford, R.P., 1954. Fatigue of rubber with two-way stretching. Rubber World 131, 365.

Gent, A.N., 1990. Cavitation in rubber: a cautionary tale. Rubber Chem. Technol. 63, G49-G53.

Gent, A.N., Lindley, P.B., Thomas, A.G., 1964. Cut growth and fatigue of rubbers. I. The relationship between cut growth and fatigue. J. Appl. Polym. Sci. 8, 455-466.

Green, A.E., Adkins, J.E., 1960. Large Elastic Deformations. The Clarendon Press, Oxford.

Greensmith, H., 1963. Rupture of rubber. X. The change in stored energy on making a small cut in a test piece held in simple extension. J. Polym. Sci. 7, 993-1002.

Griffith, A., 1920. The phenomena of rupture and flow in solids. Philos. Trans. R. Soc. London, Ser. A 221, $163-198$.

Gross, D., Kolling, S., Mueller, R., Schmidt, I., 2003. Configurational forces and their application in solid mechanics. Eur. J. Mech. Solids A/Solids 22, 669-692.

Gurtin, M.E., 2000. Configurational Forces as Basic Concept of Continuum Physics. Springer, Berlin.

Hainsworth, S.V., 2007. An environmental scanning electron microscopy investigation of fatigue crack initiation and propagation in elastomers. Polym. Test. 26, 60-70.

Holzapfel, G.A., 2000. Nonlinear Solid Mechanics. A Continuum Approach for Engineering. Wiley, Chichester.

Kienzler, R., Herrmann, G., 1997. On the properties of the Eshelby tensor. Acta Mech. 125, 73-91.

Kienzler, R., Herrmann, G., 2000. Mechanics in Material Space. Springer, Berlin.

Kienzler, R., Herrmann, G., 2002. Fracture criteria based on local properties of the Eshelby tensor. Mech. Res. Commun. 29, 521-527.

Kim, T.W., Jeong, H.Y., Choe, J.H., Kim, Y.H., 2005. Prediction of the fatigue life of tires using ced and vcct. Key Eng. Mat. 297-300, $102-107$.

Kolling, S., Mueller, R., Gross, D., 2003. A computational concept for the kinetics of defects in anisotropic materials. Comput. Mat. Sci. $26,87-94$.

Le Cam, J.-B., 2005. Endommagement en fatigue des élastomères. Ph.D. Thesis, Ecole Centrale de Nantes, France.

Le Cam, J.-B., Huneau, B., Verron, E., Gornet, L., 2004. Mechanism of fatigue crack growth in carbon black filled natural rubber. Macromolecules 37, 5011-5017.

Legorju-Jago, K., Bathias, C., 2002. Fatigue initiation and propagation in natural and synthetic rubbers. Int. J. Fatigue 24, 85-92.

Lemaitre, J., 2001. Petite histoire de l'expérimentation en mécanique des solides. Meccanica 36, 13-35.

Liebe, T., Denzer, R., Steinmann, P., 2003. Application of the material force method to isotropic continuum damage. Comput. Mech. 30, 171-184.

Lindley, P., 1972. Energy for crack growth in model rubber components. J. Strain Anal. 7, 132-140.

Mars, W.V., 2001. Multiaxial fatigue of rubber. Ph.D. Thesis, University of Toledo.

Mars, W.V., 2002. Cracking energy density as a predictor of fatigue life under multiaxial conditions. Rubber Chem. Technol. 75, 1-17.

Mars, W.V., Fatemi, A., 2002. A literature survey on fatigue analysis approaches for rubber. Int. J. Fatigue 24, 949-961.

Martinovs, A., Gonca, V., 2006. Method of forecasting of mechanical properties and durations of service life of details from rubber. in: 5th International DAAAM Baltic Conference "Industrial Engineering. Adding Innovation Capacity of Labour Force and Entrepreneurs".

Maugin, G.A., 1993. Material Inhomogeneities in Elasticity. Chapman and Hall, London.

Maugin, G.A., 1995. Material forces: concepts and applications. Appl. Mech. Rev. 48, 213-245.

Maugin, G.A., 1999. The Thermodynamics of Nonlinear Irreversible Behaviors: an Introduction, Series A, vol. 27. World Scientific Series on Nonlinear Science, World Scientific, Singapore.

Maugin, G.A., 2002. Material mechanics of materials. Theor. Appl. Mech. 27, 1-12.

Maugin, G.A., Epstein, M., Trimarco, C., 1992. Pseudomomentum and material forces in inhomogeneous materials: application to the fracture of electromagnetic materials in electromagnetoelastic fields. Int. J. Solids Struct. 29, 1889-1900.

Menzel, A., Denzer, R., Steinmann, P., 2004. On the comparison of two approaches to compute material forces for inelastic materials application to single-slip crystal-plasticity. Comput. Meth. Appl. Mech. and Eng. 193, 5411-5428.

Nguyen, T.D., Govindjee, S., Klein, P.A., Gao, H., 2005. A material force method for inelastic fracture mechanics. J. Mech. Phys. Solids 53, 91-121.

Ogden, R.W., 1972. Large deformation isotropic elasticity - on the correlation of theory and experiment for incompressible rubberlike solids. Proc. R. Soc. London 326, 565-584.

Ostoja-Kuczynski, E., Charrier, P., Verron, E., Marckmann, G., Gornet, L., Chagnon, G., 2003. Crack initiation in filled natural rubber: experimental database and macroscopic observations. In: Busfield, J., Muhr, A. (Eds.), Constitutive Models for Rubber III. A.A. Balkema, Rotterdam, pp. 3-10.

Rajagopal, K.R., Srinivasa, A.R., 2005. On the role of the Eshelby energy-momentum tensor in materials with multiple natural configurations. Math. Mech. Solids 10, 3-24.

Rice, J.R., 1968. A path independent integral and the approximate analysis of strain concentration by notches and cracks. J. Appl. Mech. 35, 379-386.

Rivlin, R.S., Thomas, A.G., 1953. Rupture of rubber. I. Characteristic energy for tearing. J. Polym. Sci. 10, $291-318$. 
Ro, H.S., 1989. Modeling and interpretation of fatigue failure initiation in rubber related to pneumatic tires. Ph.D. Thesis, Purdue University, USA.

Roach, J.F., 1982. Crack growth in elastomers under biaxial stresses. Ph.D. Thesis, University of Akron, USA.

Roberts, B.J., Benzies, J.B., 1977. The relationship between uniaxial and equibiaxial fatigue in gum and carbon black filled vulcanizates. in: Proceedings of Rubbercon'77, vol. 2.1, pp. 1-13.

Saintier, N., André, N., Cailletaud, G., Piques, R., 2006. Crack nucleation and propagation under multiaxial fatigue in natural rubber. Int. J. Fatigue 28, 61-72.

Steinmann, P., 2000. Application of material forces to hyperelastostatic fracture mechanics. I. Continuum mechanical setting. Int. J. Solids Struct. 37, 7371-7391.

Steinmann, P., 2002. On spatial and material settings of hyperelastostatic crystal defects. J. Mech. Phys. Solids 50, $1743-1766$.

Steinmann, P., Ackermann, D., Barth, F.J., 2001. Application of material forces to hyperelastostatic fracture mechanics. II. Computational setting. Int. J. Solids Struct. 38, 5509-5526.

Treloar, L.R.G., 1944. Stress-strain data for vulcanised rubber under various types of deformation. Trans. Faraday Soc. 40, 59-70.

Truesdell, C., Noll, W., 1965. Handbuch der Physik Bd. vol. III/3, The Nonlinear Field Theories of Mechanics. Springer, Berlin.

Verron, E., 1997. Contribution expérimentale et numérique aux procédés de moulage par soufflage et de thermoformage. Ph.D. Thesis, Ecole Centrale de Nantes, France.

Wöhler, A., 1867. Wöhler's experiments on the strength of metals. Engineering 2, 160-161.

Young, D., 1990. Application of fatigue methods based on fracture mechanics for tire compound development. Rubber Chem. Technol. 63, 567-581.

Zine, A., Benseddiq, N., Abdelaziz, M.N., Hocine, N.A., Bouami, D., 2006. Prediction of rubber fatigue life under multiaxial loading. Fatigue Fract. Eng. Mater. Struct. 29, 267-278. 